MATHEMATICS OF COMPUTATION

Volume 68, Number 226, April 1999, Pages 519-546

S 0025-5718(99)01026-1

\title{
THE DISCRETE PLATEAU PROBLEM: CONVERGENCE RESULTS
}

\author{
GERHARD DZIUK AND JOHN E. HUTCHINSON
}

\begin{abstract}
We solve the problem of finding and justifying an optimal fully discrete finite element procedure for approximating minimal, including unstable, surfaces. In a previous paper we introduced the general framework and some preliminary estimates, developed the algorithm and give the numerical results. In this paper we prove the convergence estimate.
\end{abstract}

\section{INTRODUCTION}

We recall from [DH4] that a minimal surface or solution of the Plateau Problem can be characterised in a number of different ways. For our purposes it is convenient to begin with the following formulation, which we restate more precisely later, cf. (3).

Let $D$ be the unit disc in $\Re^{2}$ and $\Gamma$ a smooth Jordan curve in $\Re^{n}$. Let $\mathcal{F}$ be the class of harmonic maps $u: \bar{D} \rightarrow \Re^{n}$ such that $\left.u\right|_{\partial D}: \partial D \rightarrow \Gamma$ is monotone and satisfies a certain integral "three-point condition", cf. (1). The function $u \in \mathcal{F}$ is said to be a minimal surface if $u$ is stationary in $\mathcal{F}$ for the Dirichlet energy $\mathcal{D}(u)=\frac{1}{2} \int_{D}|\nabla u|^{2}$. Such maps $u$ provide an harmonic conformal parametrisation of the corresponding minimal surface.

Let $D_{h}$ be a quasi-uniform triangulation of $D$ with grid size controlled by $h$. Let $\mathcal{F}_{h}$ be the class of discrete harmonic maps $u_{h}: \bar{D}_{h} \rightarrow \Re^{n}$ for which $u_{h}\left(\phi_{j}\right) \in \Gamma$ whenever $\phi_{j}$ is a boundary node of $D_{h}$, and which satisfy an analogue of the previous integral "three-point condition". Note that we do not require "monotonicity" of $\left.u_{h}\right|_{\partial D_{h}}$. The function $u_{h} \in \mathcal{F}_{h}$ is said to be a discrete minimal surface if $u_{h}$ is stationary within $\mathcal{F}_{h}$ for the Dirichlet energy $\mathcal{D}\left(u_{h}\right)=\frac{1}{2} \int_{D_{h}}\left|\nabla u_{h}\right|^{2}$; cf. (27) for the precise formulation.

The main result (Theorem 5.5) is that if $u$ is a nondegenerate minimal surface spanning $\Gamma$ then there exist discrete minimal surfaces $u_{h}$, unique in a ball of "almost" constant radius $\epsilon_{0}|\log h|^{-1}$, such that $\left\|u-u_{h}\right\|_{H^{1}\left(D_{h}\right)} \leq c h$, where $c$ depends on $\Gamma$ and the nondegeneracy constant $\lambda$ for $u$ but is independent of $h$. Recall that nondegeneracy corresponds to the absence of zero eigenvalues for the second variation of the Dirichlet energy at $u$, and generically corresponds to the absence of branch points. The constant $c$ blows up as $\lambda \rightarrow 0$, and this is consistent with the numerical results in [DH4]. We give an outline of the proof of the main result in Section 2.3.

Received by the editor August 26, 1996.

1991 Mathematics Subject Classification. Primary 65N30; Secondary 49Q05, 53A10.

Key words and phrases. Minimal surface, finite elements, order of convergence, Plateau Problem. 


\section{Formulation of the PROBlem}

2.1. The energy functional. We first recall some notation from [DH4] Section 3 , to which we refer for further discussion. See Section 3 of the present paper for the properties of $H^{1 / 2}$.

Let $D$ be the open unit disc in $\Re^{2}$, with boundary $\partial D$. It will be convenient to let $S^{1}$ denote another, distinct, copy of the unit circle. Let $\Gamma$ be a Jordan curve in $\Re^{n}$ with regular $C^{r}$-parametrisation $\gamma: S^{1} \rightarrow \Gamma$ where $r \geq 3$.

For $f: \partial D \rightarrow \Re^{n}$ we denote by $\Phi(f): \bar{D} \rightarrow \Re^{n}$ its unique harmonic extension to $D$ specified by

$$
\Delta \Phi(f)=0 \quad \text { in } D, \quad \Phi(f)=f \quad \text { on } \partial D .
$$

Then $\Phi: H^{1 / 2}\left(\partial D, \Re^{n}\right) \rightarrow H^{1}\left(D, \mathbb{R}^{n}\right)$ is a bounded linear map with bounded inverse.

Harmonic maps are uniquely determined by the associated boundary maps. We will use the Hilbert space $H$ of functions defined by

$$
H=\left\{\xi:\left.\partial D \rightarrow \operatorname{Re}|| \xi\right|_{H^{1 / 2}}<\infty \text { and (1) is satisfied }\right\}
$$

where

$$
\int_{0}^{2 \pi} \xi(\phi) d \phi=0, \quad \int_{0}^{2 \pi} \xi(\phi) \cos \phi d \phi=0, \quad \int_{0}^{2 \pi} \xi(\phi) \sin \phi d \phi=0 .
$$

The norm on $H$ is the usual norm $\|\cdot\|_{H^{1 / 2}}$. The corresponding affine space of maps $s: \partial D \rightarrow S^{1}$ such that $s(\phi)=\phi+\sigma(\phi)$ for some $\sigma \in H$ is denoted by $\mathcal{H}$. We also need the Banach space $T$ defined by $T=H \cap C^{0}(\partial D, \Re)$ with norm $\|\xi\|_{T}=\|\xi\|_{H^{1 / 2}}+\|\xi\|_{C^{0}}$. The corresponding affine space $\mathcal{T}$ is defined by $\mathcal{T}=$ $\mathcal{H} \cap C^{0}\left(\partial D, S^{1}\right)$. With some abuse of standard notation, we write $\|s\|=1+\|\sigma\|$ for various norms on $\sigma$.

The energy functional $E$ is defined on $\mathcal{H}$ by

$$
E(s)=\frac{1}{2} \int_{D}|\nabla \Phi(\gamma \circ s)|^{2}=\mathcal{D}(\Phi(\gamma \circ s)) .
$$

Finiteness of $E$ follows from (8). We say the harmonic function $u=\Phi(\gamma \circ s)$ is $a$ minimal surface spanning $\Gamma$, or a solution of the Plateau Problem for $\Gamma$, if and only if $s$ is monotone and stationary for $E$, i.e.

$$
\left\langle E^{\prime}(s), \xi\right\rangle=0 \quad \forall \xi \in T .
$$

As discussed in [DH4], this is equivalent to other formulations of the notion of a minimal surface.

For the proof of asymptotic convergence of the numerical method we need the following regularity result, which follows from standard regularity results (cf. [DH4] Theorem 3.2) and the Implicit Function Theorem used to write $\Gamma$ locally as the graph of a $C^{k, \alpha}$ function.

Proposition 2.1. If $\gamma \in C^{k, \alpha}$ where $k \geq 1$ and $0<\alpha<1$, and $s \in \mathcal{T}$ is monotone and stationary for $E$, then

$$
\|s\|_{C^{k, \alpha}} \leq c=c\left(\|\gamma\|_{C^{k, \alpha}},\left\|\left|\gamma^{\prime}\right|^{-1}\right\|_{L^{\infty}}\right)
$$

We next recall some properties of the energy functional from [DH4] Section 3.3. Using the notation

$$
u=\Phi(\gamma \circ s), \quad v=\Phi\left(\gamma^{\prime} \circ s \xi\right), \quad w=\Phi\left(\gamma^{\prime \prime} \circ s \xi^{2}\right),
$$


we get by formal computation

$$
\begin{aligned}
E(s) & =\frac{1}{2} \int_{D}|\nabla u|^{2} \\
\left\langle E^{\prime}(s), \xi\right\rangle & =\left.\frac{d}{d t}\right|_{t=0} E(s+t \xi)=\int_{D} \nabla u \nabla v \\
E^{\prime \prime}(s)(\xi, \xi) & =\left.\frac{d^{2}}{d t^{2}}\right|_{t=0} E(s+t \xi)=\int_{D} \nabla u \nabla w+\int_{D}|\nabla v|^{2},
\end{aligned}
$$

with an analogous expression for $E^{\prime \prime}(s)(\xi, \eta)$ obtained by bilinearity in the case of distinct variations.

If $\gamma \in C^{3}$ then $E \in C^{2}(\mathcal{T}, \Re)$, and the Fréchet derivatives are given by (6) and (7). The functional $E$ is not differentiable on $\mathcal{H}$, but if $\gamma$ and $s$ are as smooth as is necessary for the following estimates, then one has

$$
\begin{aligned}
E(s) & \leq c\|\gamma\|_{C^{1}}^{2}\|s\|_{H^{1 / 2}}^{2} \\
\left|\left\langle E^{\prime}(s), \xi\right\rangle\right| & \leq c\|\gamma\|_{C^{2}}^{2}\|s\|_{C^{1}}^{2}\|\xi\|_{H^{1 / 2}} \\
\left|E^{\prime \prime}(s)(\xi, \eta)\right| & \leq c\|\gamma\|_{C^{2}}^{2}\|s\|_{C^{1}}^{2}\|\xi\|_{H^{1 / 2}}\|\eta\|_{H^{1 / 2}}
\end{aligned}
$$

In particular, these will be used in case $s$ is stationary for $E$.

It will be important to consider the behaviour of the second derivatives of $E$ near a stationary point $s \in \mathcal{T}$. The second derivative $E^{\prime \prime}(s)$ can then be interpreted as a self-adjoint bounded map $\nabla^{2} E(s): H \rightarrow H$. Let

$$
H=H^{-} \oplus H^{0} \oplus H^{+}, \quad \xi=\xi^{-}+\xi^{0}+\xi^{+} \quad \text { if } \xi \in H,
$$

be the orthogonal decomposition generated by the eigenfunctions of $\nabla^{2} E(s)$ having negative, zero and positive eigenvalues respectively.

Proposition 2.2. Suppose $\gamma \in C^{3, \alpha}$. Suppose $s$ is monotone and stationary for $E$. Then $H^{-}$and $H^{0}$ are finite dimensional. If $\xi \in H^{-} \oplus H^{0}$ then $\xi \in H^{3 / 2}(\partial D)$ and

$$
\|\xi\|_{H^{3 / 2}} \leq \nu\|\xi\|_{H^{1 / 2}}
$$

with $\nu=\nu\left(\|\gamma\|_{C^{3, \alpha}},\left\|\left|\gamma^{\prime}\right|^{-1}\right\|_{L^{\infty}}\right)$.

Proof. The finite dimensionality of $\mathrm{H}^{-}$and $\mathrm{H}^{0}$ follow from elliptic theory; see the proof of [St, Proposition II.5.6]. It is also shown there, with a different notation, that

$$
\begin{aligned}
\left\|\Phi\left(\gamma^{\prime} \circ s \xi\right)\right\|_{H^{2}(D)} & \leq c\left(\|\gamma\|_{C^{3}},\left\|\left|\gamma^{\prime}\right|^{-1}\right\|_{L^{\infty}},\|s\|_{C^{3}},\|\xi\|_{H^{1 / 2}}\right) \\
& \leq c\left(\|\gamma\|_{C^{3, \alpha}},\left\|\left|\gamma^{\prime}\right|^{-1}\right\|_{L^{\infty}},\|\xi\|_{H^{1 / 2}}\right)
\end{aligned}
$$

by Proposition 2.1. But

$$
\begin{aligned}
\|\xi\|_{H^{3 / 2}} & \leq\left\|\frac{\gamma^{\prime} \circ s}{\left|\gamma^{\prime} \circ s\right|^{2}}\right\|_{C^{2}}\left\|\gamma^{\prime} \circ s \xi\right\|_{H^{3 / 2}} \quad \text { by }(52) \\
& \leq c\left(\|\gamma\|_{C^{3}},\left\|\left|\gamma^{\prime}\right|^{-1}\right\|_{L^{\infty}}\right)\left\|\Phi\left(\gamma^{\prime} \circ s \xi\right)\right\|_{H^{2}(D)} \quad \text { from (52) and (40). }
\end{aligned}
$$

Hence

$$
\|\xi\|_{H^{3 / 2}} \leq c\left(\|\gamma\|_{C^{3, \alpha}},\left\|\left|\gamma^{\prime}\right|^{-1}\right\|_{L^{\infty}},\|\xi\|_{H^{1 / 2}}\right),
$$

and so the required result follows by scaling. 
Higher regularity on $\gamma$ implies higher regularity on $\xi$. In particular, $\gamma \in C^{4, \alpha}$ implies $\xi \in H^{5 / 2}(\partial D)$; see the proof of [St, Proposition II.5.6].

If $s$ is monotone and stationary for $E$, we say $s$ is nondegenerate if $H^{0}=\{0\}$. The corresponding minimal surface $u=\Phi(\gamma \circ s)$ is also said to be nondegenerate. If $s$ is nondegenerate it follows that there exists a $\lambda>0$ such that for $\xi \in H$,

$$
E^{\prime \prime}(s)\left(\xi, \xi^{+}-\xi^{-}\right)=E^{\prime \prime}(s)\left(\xi^{+}, \xi^{+}\right)-E^{\prime \prime}(s)\left(\xi^{-}, \xi^{-}\right) \geq \lambda\|\xi\|_{H^{1 / 2}}^{2}
$$

We call $\lambda$ the nondegeneracy constant for $s$.

2.2. The discrete energy functional. We recall the necessary notation from [DH4] Section 4 and prove some preliminary estimates. Let

Let $\mathcal{G}_{h}$ be a quasi-uniform triangulation of $D$ with grid size comparable to $h$.

$$
\begin{aligned}
D_{h} & =\bigcup\left\{G \mid G \in \mathcal{G}_{h}\right\}, \\
\partial D_{h} & =\bigcup\left\{E_{j} \mid 1 \leq j \leq M\right\} \quad \text { where the } E_{j} \text { are the boundary edges, }
\end{aligned}
$$

and let

$$
\mathcal{B}_{h}=\left\{\phi_{1}, \ldots, \phi_{M}\right\} \quad \text { be the set of boundary nodes. }
$$

The projection $\pi: \partial D \rightarrow \partial D_{h}$ is defined by

$$
\pi\left(e^{i\left((1-t) \phi_{j}+t \phi_{j+1}\right)}\right)=(1-t) e^{i \phi_{j}}+t e^{i \phi_{j+1}}
$$

for $0 \leq t \leq 1,1 \leq j \leq M$.

In order to have a discrete analogue $E_{h}$ of the functional $E$ we define the following discrete analogues of $H^{1}\left(D ; \Re^{n}\right), H^{1 / 2}\left(\partial D ; \Re^{n}\right), H, T, \mathcal{H}$ and $\mathcal{T}$ :

$$
\begin{aligned}
& X_{h}^{n}=\left\{u_{h} \in C^{0}\left(D_{h} ; \Re^{n}\right) \mid u_{h} \in P_{1}(G) \text { for } G \in \mathcal{G}_{h}\right\} \\
& x_{h}^{n}=\left\{f_{h} \in C^{0}\left(\partial D_{h} ; \Re^{n}\right) \mid f_{h} \in P_{1}\left(E_{j}\right) \text { for } 1 \leq j \leq M\right\} \\
& H_{h}=\left\{\xi_{h} \in C^{0}(\partial D ; \Re) \mid \xi_{h} \in P_{1}\left(\pi^{-1}\left(E_{j}\right)\right) \text { if } 1 \leq j \leq M, \xi_{h} \text { satisfies }(1)\right\} \\
& \mathcal{H}_{h}=\left\{s_{h} \in C^{0}\left(\partial D ; S^{1}\right) \mid s_{h}(\phi)=\phi+\sigma_{h}(\phi) \text { for some } \sigma_{h} \in H_{h}\right\}
\end{aligned}
$$

Thus $H_{h} \subset T \subset H, \mathcal{H}_{h} \subset \mathcal{T} \subset \mathcal{H}$, and the space of variations at any $s_{h} \in \mathcal{H}_{h}$ is naturally identified with $H_{h}$. Setting $n=1$, we similarly define $X_{h}$ and $x_{h}$.

We will make frequent use of the following inverse-type estimates:

Proposition 2.3. If $\xi_{h} \in H_{h}$ then

$$
\begin{aligned}
\left\|\xi_{h}\right\|_{H^{1}} & \leq c h^{-1 / 2}\left\|\xi_{h}\right\|_{H^{1 / 2}} \\
\left\|\xi_{h}\right\|_{C^{0}} & \leq c|\log h|^{1 / 2}\left\|\xi_{h}\right\|_{H^{1 / 2}}
\end{aligned}
$$

Proof. The first estimate is standard. The second is shown in [DH1] Proposition 5.3 .

Suppose $f \in C^{0}\left(\partial D ; \Re^{n}\right)$. One defines the "linear interpolants"

$$
\begin{aligned}
I_{h} f \in x_{h}^{n}, \quad I_{h} f\left((1-t) e^{i \phi_{j}}+t e^{i \phi_{j+1}}\right)=(1-t) f\left(e^{i \phi_{j}}\right)+t f\left(e^{i \phi_{j+1}}\right), \\
I_{h}^{\partial D} f \in C^{0}\left(\partial D ; \Re^{n}\right), \quad I_{h} f\left((1-t) e^{i \phi_{j}}+t e^{i \phi_{j+1}}\right)=(1-t) f\left(e^{i \phi_{j}}\right)+t f\left(e^{i \phi_{j+1}}\right),
\end{aligned}
$$


where $0 \leq t \leq 1,1 \leq j \leq M$. Here and elsewhere, $\phi_{M+1}=\phi_{1}$. Note the different domains of $I_{h} f$ and $I_{h}^{\partial D} f$. Note also that the image of $I_{h}(\gamma \circ s)$ is a polygonal approximation to $\Gamma$, and $I_{h}(\gamma \circ s)\left(\phi_{j}\right)=\gamma \circ s\left(\phi_{j}\right) \in \Gamma$ for $\phi_{j} \in \mathcal{B}_{h}$. Finally,

$$
I_{h}^{\partial D} f=I_{h} f \circ \pi \text {. }
$$

Another type of approximation operator we require is a map $p_{h}: T(\mathcal{T}) \rightarrow$ $H_{h}\left(\mathcal{H}_{h}\right)$. The usual interpolation operator does not preserve the normalisation conditions (1). However, if we first interpolate and then project onto $H_{h}$, the resulting operator still satisfies all the usual estimates. The proof of the following is essentially given in [DH1] Proposition 5.2.

Proposition 2.4. There is a bounded linear operator $p_{h}: T \rightarrow H_{h}$ such that, in particular,

$$
\left\|\xi-p_{h} \xi\right\|_{H^{s}} \leq c h^{k-s}\|\xi\|_{H^{k}}
$$

for $s=0, \frac{1}{2}, 1$ and $k=1, \frac{3}{2}, 2$. Moreover,

$$
\begin{array}{ll}
\left\|\xi-p_{h} \xi\right\|_{C^{0,1}} \leq c h\|\xi\|_{C^{2}}, & \left\|p_{h} \xi\right\|_{C^{0,1}} \leq c\|\xi\|_{C^{0,1}} \\
\left\|\xi-p_{h} \xi\right\|_{C^{0}} \leq c h^{2}\|\xi\|_{C^{2}}, & \left\|\xi-p_{h} \xi\right\|_{C^{0}} \leq c h\|\xi\|_{C^{1}}
\end{array}
$$

If $s \in \mathcal{T}$ and $s(\phi)=\phi+\sigma(\phi)$, then $p_{h} s$ is defined by $p_{h} s(\phi)=\phi+p_{h} \sigma(\phi)$, $s-p_{h} s:=\sigma-p_{h} \sigma$, and hence $p_{h} s$ satisfies estimates similar to those for $p_{h} \xi$.

For $f_{h} \in x_{h}$ the discrete harmonic extension $\Phi_{h} f_{h} \in X_{h}$ is defined by

$$
\triangle_{h} \Phi_{h} f_{h}=0 \quad \text { in } \quad D_{h}, \quad \Phi_{h} f_{h}=f_{h} \quad \text { on } \partial D_{h},
$$

where $\triangle_{h}$ is the discrete Laplacian and the first equation in (25) is interpreted as $\int_{D_{h}} \nabla\left(\Phi_{h} f_{h}\right) \nabla \psi_{h}=0$ for all $\psi_{h} \in X_{h}$ such that $\psi_{h}=0$ on $\partial D_{h}$. If $f_{h} \in x_{h}^{n}$ the discrete harmonic extension $\Phi_{h} f_{h}$ is defined componentwise.

For $s_{h} \in \mathcal{H}_{h}$, the discrete energy functional $E_{h}$ is defined by

$$
E_{h}\left(s_{h}\right)=\frac{1}{2} \int_{D_{h}}\left|\nabla \Phi_{h} I_{h}\left(\gamma \circ s_{h}\right)\right|^{2}=\mathcal{D}_{h}\left(\Phi_{h} I_{h}\left(\gamma \circ s_{h}\right)\right) .
$$

Note that $E_{h}$ is of course not the restriction of $E$ to $\mathcal{H}_{h}$. The discrete harmonic function $u_{h}=\Phi_{h} I_{h}\left(\gamma \circ s_{h}\right)$ is said to be a discrete minimal surface spanning $\Gamma$, or a solution of the discrete Plateau Problem for $\Gamma$, if and only if

$$
\left\langle E_{h}^{\prime}\left(s_{h}\right), \xi_{h}\right\rangle=0 \quad \forall \xi_{h} \in H_{h} .
$$

Note that we do not require monotonicity of $s_{h}$, as is the case for $s$ in (3).

The derivatives of $E_{h}$, cf. (5)-(7), are given by

$$
\begin{aligned}
E_{h}\left(s_{h}\right) & =\frac{1}{2} \int_{D_{h}}\left|\nabla u_{h}\right|^{2}, \\
\left\langle E_{h}^{\prime}\left(s_{h}\right), \xi_{h}\right\rangle & =\int_{D_{h}} \nabla u_{h} \nabla v_{h}, \\
E_{h}^{\prime \prime}\left(s_{h}\right)\left(\xi_{h}, \xi_{h}\right) & =\int_{D_{h}} \nabla u_{h} \nabla w_{h}+\int_{D_{h}}\left|\nabla v_{h}\right|^{2},
\end{aligned}
$$

where

$$
u_{h}=\Phi_{h} I_{h}\left(\gamma \circ s_{h}\right), \quad v_{h}=\Phi_{h} I_{h}\left(\gamma^{\prime} \circ s_{h} \xi_{h}\right), \quad w_{h}=\Phi_{h} I_{h}\left(\gamma^{\prime \prime} \circ s_{h} \xi_{h}^{2}\right) .
$$


For $\xi_{h} \in H_{h}$ and $s$ stationary for $E$, we define the projection of the decomposition (11):

$$
\xi_{h}^{(-)}=p_{h} \xi_{h}^{-}, \quad \xi_{h}^{(0)}=p_{h} \xi_{h}^{0}, \quad \xi_{h}^{(+)}=p_{h} \xi_{h}^{+} .
$$

Note that $\xi_{h}^{-}, \xi_{h}^{0}$ and $\xi_{h}^{+}$do not normally belong to $H_{h}$; in particular, the first two are smooth functions. However, if $H^{-}=H^{0}=\{0\}$ then $\xi_{h}=\xi_{h}^{+}=\xi_{h}^{(+)}$.

Proposition 2.5. If $\xi_{h} \in H_{h}$ then

$$
\xi_{h}=\xi_{h}^{(-)}+\xi_{h}^{(0)}+\xi_{h}^{(+)}
$$

and $\xi_{h}^{(-)}, \xi_{h}^{(0)}, \xi_{h}^{(+)} \in H_{h}$. Moreover, with $\nu$ as in (12),

$$
\begin{aligned}
\left\|\xi_{h}^{-}-\xi_{h}^{(-)}\right\|_{H^{1 / 2}} & \leq \operatorname{ch} \nu\left\|\xi_{h}\right\|_{H^{1 / 2}}, \quad\left\|\xi_{h}^{-}-\xi_{h}^{(-)}\right\|_{H^{1}} \leq c h^{1 / 2} \nu\left\|\xi_{h}\right\|_{H^{1 / 2}}, \\
\left\|\xi_{h}^{0}-\xi_{h}^{(0)}\right\|_{H^{1 / 2}} & \leq \operatorname{ch} \nu\left\|\xi_{h}\right\|_{H^{1 / 2}}, \quad\left\|\xi_{h}^{0}-\xi_{h}^{(0)}\right\|_{H^{1}} \leq h^{1 / 2} \nu\left\|\xi_{h}\right\|_{H^{1 / 2}}, \\
\left\|\xi_{h}^{+}-\xi_{h}^{(+)}\right\|_{H^{1 / 2}} & \leq \operatorname{ch} \nu\left\|\xi_{h}\right\|_{H^{1 / 2}}, \quad\left\|\xi_{h}^{+}-\xi_{h}^{(+)}\right\|_{H^{1}} \leq c h^{1 / 2} \nu\left\|\xi_{h}\right\|_{H^{1 / 2}} .
\end{aligned}
$$

Proof. Since $p_{h} \xi_{h}=\xi_{h}$, (33) follows from (11). Next, from (22) and (12),

$$
\left\|\xi_{h}^{-}-\xi_{h}^{(-)}\right\|_{H^{1 / 2}}=\left\|\xi_{h}^{-}-p_{h} \xi_{h}^{-}\right\|_{H^{1 / 2}} \leq \operatorname{ch}\left\|\xi_{h}^{-}\right\|_{H^{3 / 2}} \leq \operatorname{ch} \nu\left\|\xi_{h}^{-}\right\|_{H^{1 / 2}} .
$$

The proof of the other inequality in (34), and of (35), is similar.

For (36) just note that, from (33) and (11),

$$
\xi_{h}^{+}-\xi_{h}^{(+)}=-\left(\xi_{h}^{-}-\xi_{h}^{(-)}\right)-\left(\xi_{h}^{0}-\xi_{h}^{(0)}\right),
$$

and use the previous estimates.

We remark that the decomposition (33), unlike (11), is not an orthogonal decomposition. We also note that the powers of $h$ in (34)-(36) can be increased by $1 / 2$ if we assume $\|\xi\|_{H^{2}} \leq \nu\|\xi\|_{H^{1 / 2}}$; this latter holds if $\gamma \in C^{4, \alpha}$, cf. the remarks after Proposition 2.2.

2.3. Structure of the proof. The main error estimates, Theorems 5.4 and 5.5, are proved in Section 5. To motivate our approach and the need for the various preliminary estimates in Sections 4 and 5, we outline in an informal manner the structure of the proof of Theorem 5.4. See Figure 1.

Assume $s$ is a nondegenerate stationary point for $E$ with nondegeneracy constant $\lambda$. One applies the Inverse Function Theorem to the derivative $E_{h}^{\prime}: \mathcal{H}_{h} \rightarrow H_{h}^{\prime}$ (the dual space of $H_{h}$ ) in a neighbourhood of the point $p_{h} s$; remember that $H_{h}$ is the tangent space for $\mathcal{H}_{h}$, i.e. the space of variations at any $t_{h} \in \mathcal{H}_{h}$. Identify $E_{h}^{\prime \prime}$ with the derivative $\left(E_{h}^{\prime}\right)^{\prime}$ of $E_{h}^{\prime}$; thus $\left(E_{h}^{\prime}\right)^{\prime}\left(t_{h}\right): H_{h} \rightarrow H_{h}^{\prime}$ for $t_{h} \in \mathcal{H}_{h}$.

There are three estimates to be proved.

A: $\left\|E_{h}^{\prime}\left(p_{h} s\right)\right\| \leq c_{1} h$ for $h$ sufficiently small. This follows immediately from the consistency estimate (Proposition 4.2) for the first derivative. See (109).

$\mathbf{B}_{1}:\left\|\left\langle\left(E_{h}^{\prime}\right)^{\prime}\left(p_{h} s\right), \xi_{h}\right\rangle\right\| \geq \frac{\lambda}{2}\left\|\xi_{h}\right\|$ for $h$ sufficiently small; i.e. "the slope of $E_{h}^{\prime}$ at $p_{h} s$ is bounded away from 0 by $\lambda / 2$ ". This is a nondegeneracy estimate on $E_{h}^{\prime \prime}\left(p_{h} s\right)$ and is established in Proposition 5.3, using the one-sided consistency estimate (Proposition 4.3) for the second derivative, and the regularity of members of the negative eigenspaces to compensate for the one-sided nature of that estimate. See (114).

$\mathbf{B}_{2}:\left\|\left(E_{h}^{\prime}\right)^{\prime}\left(p_{h} s\right)-\left(E_{h}^{\prime}\right)^{\prime}\left(p_{h} s+\eta_{h}\right)\right\| \leq \lambda / 4$ for $h$ sufficiently small and $\eta_{h}$ sufficiently close to $p_{h} s$, more precisely for $\eta_{h} \leq \epsilon_{0} /|\log h|$; i.e. "the slope of $E_{h}^{\prime}$ near $p_{h} s$ 


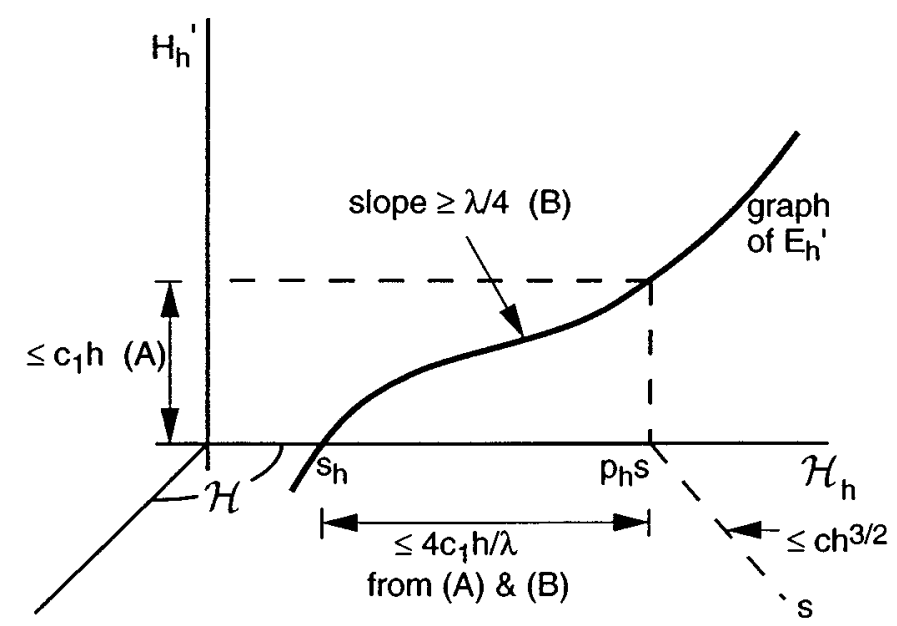

FiguRE 1

differs from that at $p_{h} s$ by at most $\lambda / 4$ ". This is a consequence of Proposition 5.2. See (115). As indicated in the diagram, it follows that there exists a unique stationary point $s_{h}$ for $E_{h}$ such that $\left\|p_{h} s-s_{h}\right\|_{H^{1 / 2}} \leq 4 c_{1} h / \lambda$, and hence such that $\left\|s-s_{h}\right\|_{H^{1 / 2}} \leq c h / \lambda$.

\section{Preliminary estimates}

One can define the $H^{s}(\partial D)$ and $H^{s}(D)$ norms for any real $s$; but apart from non-negative integers $s$ we will only need the following cases.

For $f: \partial D \rightarrow \Re$ the $H^{1 / 2}(\partial D)$ seminorm is defined by

$$
|f|_{H^{1 / 2}(\partial D)}^{2}=\int_{\partial D} \int_{\partial D} \frac{|f(\phi)-f(\bar{\phi})|^{2}}{|\phi-\bar{\phi}|^{2}} d \phi d \bar{\phi}
$$

and for $u: D \rightarrow \Re$ the $H^{1 / 2}(D)$ seminorm is defined by

$$
|u|_{H^{1 / 2}(D)}^{2}=\int_{D} \int_{D} \frac{|u(x)-u(\bar{x})|^{2}}{|x-\bar{x}|^{3}} d x d \bar{x} .
$$

In both cases the corresponding norm is given by

$$
\|\cdot\|_{H^{1 / 2}}^{2}=\|\cdot\|_{L^{2}}^{2}+|\cdot|_{H^{1 / 2}}^{2} .
$$

Also,

$$
\begin{gathered}
|f|_{H^{3 / 2}(\partial D)}=\left|f^{\prime}\right|_{H^{1 / 2}(\partial D)}, \quad|u|_{H^{3 / 2}(D)}=|\nabla u|_{H^{1 / 2}(D)}, \\
\|\cdot\|_{H^{3 / 2}}^{2}=\|\cdot\|_{L^{2}}^{2}+|\cdot|_{H^{3 / 2}}^{2} .
\end{gathered}
$$

If $u \in H^{s+1 / 2}(D)$ for $s=1 / 2,1,3 / 2$ (in fact for $s>0$ ), then $u$ has a well-defined trace $f$ on $\partial D$ and

$$
\|f\|_{H^{s}(\partial D)} \leq c\|u\|_{H^{s+1 / 2}(D)} .
$$

More precisely, the previous estimate is true for $u \in C^{\infty}(\bar{D})$, and the definition of trace is extended by continuity and density to the general case. Such an estimate is not true for smooth $u$ if $s=0$, but in this case if $\Delta u \in L^{2}(D)$ the following 
estimate holds, and then the trace operator is again defined by continuity and density to satisfy

$$
\|f\|_{L^{2}(\partial D)} \leq c\left(\|u\|_{H^{1 / 2}(D)}+\|\triangle u\|_{L^{2}(D)}\right) .
$$

See [LM], Chapter 1, Section 9.2, page 193 (remarks before Theorem 8.1) and page 187, Theorem 7.3. Note that by considering kernels it is clear that in the case $s=1 / 2$ one can replace the norms in (40) by the corresponding seminorms. We will frequently use this fact without further remark.

Conversely, if $f \in H^{s}(\partial D)$ for $s=0,1 / 2,1,3 / 2$ (with similar results for any real $s)$, then there is a unique harmonic function $\Phi(f)$ defined on $D$ with trace $f$ as before, and in particular

$$
\|\Phi(f)\|_{H^{s+1 / 2}(D)} \leq c\|f\|_{H^{s}(\partial D)} .
$$

See [LM], Theorem 7.4, page 188. Note that for $s=1 / 2,1,3 / 2$ the norms can be replaced by seminorms, as follows again by considering kernels; we will use this fact without further comment.

In the case of $L^{2}$ boundary data one also has, with $u=\Phi(f)$,

$$
\begin{aligned}
\lim _{r \rightarrow 1^{-}} u(r \theta) & =f(\theta) \quad \text { for a.e. } \theta \in \partial D, \\
\left\|\sup _{0 \leq r<1}|u(r \cdot)|\right\|_{L^{2}(\partial D)} & \leq c\|f\|_{L^{2}(\partial D) .}
\end{aligned}
$$

See [JK], Theorem (1.16), page 11. In particular, $u(r \cdot) \rightarrow f$ in $L^{2}(\partial D)$ as $r \rightarrow 1^{-}$. (The formulae (43) and (44) are established using the Poisson integral representation of $u$ from $f$. The function $u$ agrees with $\Phi(f)$ in (42), since it agrees for $f$ in the dense subset $C^{\infty}(\partial D) \subset L^{2}(\partial D)$ by the maximum principle, and since the Poisson integral map and $\Phi$ are both bounded as maps from $L^{2}(\partial D)$ into $L^{2}(D)$, for example.)

If $f: \partial D \rightarrow \Re$ has the Fourier series expansion

$$
f(\phi)=a_{0}+\sum_{n=1}^{\infty}\left(a_{n} \cos n \phi+b_{n} \sin n \phi\right),
$$

then one can define

$$
\begin{aligned}
\|f\|_{L^{2}(\partial D)}^{2} & =a_{0}^{2}+\sum_{n=1}^{\infty}\left(a_{n}^{2}+b_{n}^{2}\right), \\
|f|_{H^{s}(\partial D)}^{2} & =\sum_{n=1}^{\infty} n^{2 s}\left(a_{n}^{2}+b_{n}^{2}\right) \quad s>0, \\
\|f\|_{H^{s}(\partial D)}^{2} & =\|f\|_{L^{2}(\partial D)}^{2}+|f|_{H^{s}(\partial D)}^{2} .
\end{aligned}
$$

These norms and seminorms are equivalent to the usual definitions in case $s \in \mathbb{N}$, and the previous definitions in case $s=1 / 2$ or $3 / 2$. The harmonic extension of $f$ is given by

$$
(\Phi f)(r, \phi)=a_{0}+\sum_{n=1}^{\infty} r^{n}\left(a_{n} \cos n \phi+b_{n} \sin n \phi\right)
$$


Proposition 3.1. Suppose $f, g: \partial D \rightarrow \Re$. Then

$$
\begin{aligned}
|f g|_{H^{1 / 2}} & \leq\|f\|_{C^{0}}|g|_{H^{1 / 2}}+|f|_{H^{1 / 2}}\|g\|_{C^{0}}, \\
\|f g\|_{H^{1 / 2}} & \leq c\|f\|_{C^{0,1}}\|g\|_{H^{1 / 2}} \\
|f g|_{H^{1}} & \leq\|f\|_{C^{0}}|g|_{H^{1}}+|f|_{H^{1}}\|g\|_{C^{0}}, \\
\|f g\|_{H^{1}} & \leq c\|f\|_{C^{0,1}}\|g\|_{H^{1}} \\
\|f g\|_{H^{3 / 2}} & \leq c\|f\|_{C^{2}}\|g\|_{H^{3 / 2}} .
\end{aligned}
$$

Proof. The first two inequalities follow from (37). The next two inequalities are standard. The last inequality follows from (49).

The next proposition will be applied in case $g$ is (a component of) $\gamma, \gamma^{\prime}$ or $\gamma^{\prime \prime}$, and $s$ is either smooth or piecewise linear and continuous. In particular, $g$ will be at least $C^{1}$ but $s$ may be only $C^{0,1}$ in some cases.

Proposition 3.2. Suppose $s=i d+\sigma: \partial D \rightarrow S^{1}, g: S^{1} \rightarrow \Re$. Then

$$
\begin{aligned}
\|g \circ s\|_{C^{0}} & \leq\|g\|_{C^{0}} \leq\|g\|_{C^{0}}\|s\|_{C^{0}}, \\
\|g \circ s\|_{C^{0,1}} & \leq c\|g\|_{C^{1}}\|s\|_{C^{0,1}}, \\
\|g \circ s\|_{C^{1}} & \leq c\|g\|_{C^{1}}\|s\|_{C^{1}}, \\
\|g \circ s\|_{C^{2}} & \leq c\|g\|_{C^{2}}\|s\|_{C^{2}}^{2} \\
\|g \circ s\|_{L^{2}} & \leq c\|g\|_{C^{0}} \leq c\|g\|_{C^{0}}\|s\|_{L^{2}}, \\
\|g \circ s\|_{H^{1 / 2}} & \leq c\|g\|_{C^{1}}\|s\|_{H^{1 / 2}}, \\
\|g \circ s\|_{H^{1}} & \leq c\|g\|_{C^{1}}\|s\|_{H^{1}} .
\end{aligned}
$$

Proof. Recall that $\|s\|_{C^{0}}=1+\|\sigma\|_{C^{0}} \geq 1$. Then (53) is immediate, and similarly for (57). Also (54) is immediate. For (55), (56) and (59), note that

$$
(g \circ s)^{\prime}=g^{\prime} \circ s s^{\prime}, \quad(g \circ s)^{\prime \prime}=g^{\prime \prime} \circ s\left(s^{\prime}\right)^{2}+g^{\prime} \circ s s^{\prime \prime} .
$$

Inequality (58) follows from (37).

The following proposition will typically be applied in case $g$ is $\gamma, \gamma^{\prime}$ or $\gamma^{\prime \prime}$ and in particular is $C^{1}$; and either $s_{1}=s_{0}$ and $s_{2}=p_{h} s_{0}$, or $s_{1}=p_{h} s_{0}$ and $s_{2}=p_{h} s_{0}+\eta_{h}$ for an arbitrary $\eta_{h} \in H_{h}$. Note that in (61) only the $C^{0}$ norm of $s_{1}-s_{2}$ is required, and in case $s_{1}-s_{2} \in H_{h}$ this will be estimated by the inverse estimate (20).

Proposition 3.3. Suppose $s_{i}=i d+\sigma_{i}: \partial D \rightarrow S^{1}$ for $i=1,2$ and $g: S^{1} \rightarrow \Re$. Then

(60) $\left\|g \circ s_{1}-g \circ s_{2}\right\|_{L^{2}} \leq c|g|_{C^{1}}\left\|s_{1}-s_{2}\right\|_{L^{2}}$,

(61) $\left|g \circ s_{1}-g \circ s_{2}\right|_{H^{1 / 2}} \leq c\|g\|_{C^{2}}\left(\left\|s_{1}\right\|_{C^{0,1}}+\left\|s_{1}-s_{2}\right\|_{C^{0}}\right)\left\|s_{1}-s_{2}\right\|_{H^{1 / 2}}$,

(62) $\left|g \circ s_{1}-g \circ s_{2}\right|_{H^{1}} \leq c\|g\|_{C^{2}}\left\|s_{1}\right\|_{C^{0,1}}\left\|s_{1}-s_{2}\right\|_{H^{1}}$,

(63) $\left|g \circ s_{1}-g \circ s_{2}\right|_{C^{0,1}} \leq c\|g\|_{C^{2}}\left\|s_{1}\right\|_{C^{0,1}}\left\|s_{1}-s_{2}\right\|_{C^{0,1}}$.

Proof. The proof of (60) is immediate.

For (61) write

$$
\eta(\phi)=s_{2}(\phi)-s_{1}(\phi)
$$


Then

$$
\begin{aligned}
& \left|\left(g\left(s_{1}(\phi)\right)-g\left(s_{1}(\phi)+\eta(\phi)\right)\right)-\left(g\left(s_{1}(\bar{\phi})\right)-g\left(s_{1}(\bar{\phi})+\eta(\bar{\phi})\right)\right)\right| \\
& =\quad\left|-\int_{0}^{1} g^{\prime}\left(s_{1}(\phi)+t \eta(\phi)\right) d t \eta(\phi)+\int_{0}^{1} g^{\prime}\left(s_{1}(\bar{\phi})+t \eta(\bar{\phi})\right) d t \eta(\bar{\phi})\right| \\
& \leq \quad \int_{0}^{1}\left|g^{\prime}\left(s_{1}(\phi)+t \eta(\phi)\right)\right| d t|\eta(\phi)-\eta(\bar{\phi})| \\
& \quad+\int_{0}^{1}\left|g^{\prime}\left(s_{1}(\phi)+t \eta(\phi)\right)-g^{\prime}\left(s_{1}(\bar{\phi})+t \eta(\bar{\phi})\right)\right| d t|\eta(\bar{\phi})| \\
& \leq\|g\|_{C^{1}}|\eta(\phi)-\eta(\bar{\phi})|+\|g\|_{C^{2}}\left(\left|s_{1}(\phi)-s_{1}(\bar{\phi})\right|+|\eta(\phi)-\eta(\bar{\phi})|\right)|\eta(\bar{\phi})| .
\end{aligned}
$$

It now follows from (37) that

$$
\begin{aligned}
& \left|g \circ s_{1}-g \circ\left(s_{1}+\eta\right)\right|_{H^{1 / 2}} \\
& \quad \leq\|g\|_{C^{1}}|\eta|_{H^{1 / 2}}+\|g\|_{C^{2}}\left(c\left\|s_{1}\right\|_{C^{0,1}}\|\eta\|_{L^{2}}+|\eta|_{H^{1 / 2}}\|\eta\|_{C^{0}}\right) \\
& \quad \leq c\|g\|_{C^{2}}\left(\left\|s_{1}\right\|_{C^{0,1}}+\|\eta\|_{C^{0}}\right)\|\eta\|_{H^{1 / 2}},
\end{aligned}
$$

recalling that $\left\|s_{1}\right\|_{C^{0,1}} \geq 1$. This establishes (61).

For (62) we compute

$$
\begin{aligned}
& \left\|\left(g \circ s_{1}-g \circ s_{2}\right)^{\prime}\right\|_{L^{2}} \\
& \quad=\left\|g^{\prime} \circ s_{1} s_{1}^{\prime}-g^{\prime} \circ s_{2} s_{2}^{\prime}\right\|_{L^{2}} \\
& \quad \leq\left\|g^{\prime} \circ s_{1}-g^{\prime} \circ s_{2}\right\|_{L^{2}}\left\|s_{1}^{\prime}\right\|_{L^{\infty}}+\left\|g^{\prime} \circ s_{2}\right\|_{C^{0}}\left\|s_{1}^{\prime}-s_{2}^{\prime}\right\|_{L^{2}} \\
& \quad \leq c|g|_{C^{2}}\left\|s_{1}-s_{2}\right\|_{L^{2}}\left|s_{1}\right|_{C^{0,1}}+|g|_{C^{1}}\left|s_{1}-s_{2}\right|_{H^{1}} \quad \text { from (60) } \\
& \quad \leq c\|g\|_{C^{2}}\left\|s_{1}\right\|_{C^{0,1}}\left\|s_{1}-s_{2}\right\|_{H^{1}} .
\end{aligned}
$$

For (63) we similarly estimate $\left\|\left(g \circ s_{1}-g \circ s_{2}\right)^{\prime}\right\|_{L^{\infty}}$.

The next proposition will be used repeatedly in the consistency and non-degeneracy estimates of Sections 4 and 5, particularly in case $s=1$. In Corollary 3.5 we see that the $H^{1}\left(D_{h}\right)$ seminorm of the discrete harmonic extension of a discrete function $f$ can be estimated by the $H^{1}(D)$ seminorm of the smooth harmonic extension of the function. This is not true for general smooth $f$. The corollary is due to Bramble, Pasciak and Schatz [BPS].

Proposition 3.4. If $f \in H^{s}\left(\partial D, \Re^{n}\right)$ where $s=1,3 / 2$, then

$$
\begin{aligned}
\left|\Phi(f)-\Phi_{h} I_{h}(f)\right|_{H^{1}\left(D_{h}\right)} & \leq c h^{s-1 / 2}|f|_{H^{s}(\partial D)} \\
\left|\Phi_{h} I_{h}(f)\right|_{H^{1}\left(D_{h}\right)} & \leq|f|_{H^{1 / 2}(\partial D)}+c h^{s-1 / 2}|f|_{H^{s}(\partial D)} .
\end{aligned}
$$

Proof. It is sufficient to take $n=1$. Let

$$
u=\Phi(f), \quad u_{h}=\Phi_{h} I_{h}(f) .
$$

Note that $I_{h} f$ is well-defined, as $f \in C^{0}\left(\partial D_{h}\right)$. Since $u_{h}=I_{h} u$ on $\partial D_{h}$, it follows in the usual way from the weak form of Laplace's equation and (25) that

$$
0=\int_{D_{h}}\left(\nabla u_{h}-\nabla u\right)\left(\nabla u_{h}-\nabla I_{h} u\right),
$$

and so

$$
\int_{D_{h}}\left|\nabla u-\nabla u_{h}\right|^{2}=\int_{D_{h}}\left(\nabla u-\nabla u_{h}\right)\left(\nabla u-\nabla I_{h} u\right) .
$$


Hence

$$
\begin{aligned}
\left|u-u_{h}\right|_{H^{1}\left(D_{h}\right)} & \leq\left|u-I_{h} u\right|_{H^{1}\left(D_{h}\right)} \\
& \leq c h^{s-1 / 2}|u|_{H^{s+1 / 2}\left(D_{h}\right)} \leq c h^{s-1 / 2}|u|_{H^{s+1 / 2}(D)} \\
& \leq c h^{s-1 / 2}|u|_{H^{s}(\partial D)},
\end{aligned}
$$

from a standard interpolation result and (42). This gives (64), and (65) then follows from (42).

Corollary 3.5. Suppose $f_{h} \in H_{h}$. Then

$$
\left|\Phi_{h} I_{h}\left(f_{h}\right)\right|_{H^{1}\left(D_{h}\right)} \leq c\left|\Phi\left(f_{h}\right)\right|_{H^{1}(D)}
$$

Proof. This follows from (65) with $s=1$, the inverse estimate (19), and (40).

Proposition 3.7 will allow us to estimate various quantities involving a harmonic function and the discrete disc $D_{h}$, in terms of the trace of the harmonic function on $D$. But first we need an elementary lemma.

Lemma 3.6. Suppose $u \in H^{1}(D)$, and $\pi: \partial D \rightarrow \partial D_{h}$ is as in (14). Then

$$
\|u-u \circ \pi\|_{L^{2}(\partial D)} \leq c h|u|_{H^{1}\left(D \backslash D_{h}\right)}
$$

Proof. Let $L_{\theta}$ be the straight line segment joining $\theta \in \partial D$ to $\pi(\theta) \in \partial D_{h}$. Then

$$
|u(\theta)-u \circ \pi(\theta)|^{2} \leq\left(\int_{L_{\theta}}|\nabla u|\right)^{2} \leq c h^{2} \int_{L_{\theta}}|\nabla u|^{2} .
$$

Each $z \in D \backslash D_{h}$ can be written uniquely as $(\theta, y) \in \partial D \times \Re^{+}$, where $z \in L_{\theta}$ and $y$ is the distance of $z$ from $\theta \in \partial D$. The corresponding map $(\theta, y) \mapsto z$ has Jacobian $J$ satisfying $1-c h^{2} \leq J \leq 1$. Hence

$$
\int_{\partial D}|u-u \circ \pi|^{2} \leq c h^{2} \int_{\partial D} d \theta \int_{L_{\theta}}|\nabla u|^{2} \leq c h^{2} \int_{D \backslash D_{h}}|\nabla u|^{2} .
$$

In the following proposition and elsewhere, $\frac{\partial u}{\partial \nu}$ and $\frac{\partial u}{\partial \tau}$ denote normal and tangential derivatives on the relevant curve.

Proposition 3.7. Suppose $u$ is harmonic in $D$ with trace $\left.u\right|_{\partial D} \in L^{2}(\partial D) H^{1}(\partial D)$ as appropriate. Then

$$
\begin{aligned}
\|u\|_{L^{2}\left(D \backslash D_{h}\right)} & \leq c h\|u\|_{L^{2}(\partial D)}, \\
\|\nabla u\|_{L^{2}\left(D \backslash D_{h}\right)} & \leq c h|u|_{H^{1}(\partial D)}, \\
\|u-u \circ \pi\|_{L^{2}(\partial D)} & \leq c h^{2}|u|_{H^{1}(\partial D)}, \\
\left\|\frac{\partial u}{\partial \nu}\right\|_{L^{2}\left(\partial D_{h}\right)} & \leq c|u|_{H^{1}(\partial D)} .
\end{aligned}
$$

Proof. Let $D(\rho)$ be the disc of radius $\rho$. From (44)

$$
\|u\|_{L^{2}(\partial D(\rho))} \leq c\|u\|_{L^{2}(\partial D)} .
$$

Integrating the square of this inequality with respect to $\rho$ from $1-c h^{2}$ to 1 now establishes (70).

Since $\nabla u$ is also harmonic, it follows that

$$
\|\nabla u\|_{L^{2}\left(D \backslash D_{h}\right)} \leq \operatorname{ch}\|\nabla u\|_{L^{2}(\partial D)} .
$$


But

$$
\int_{\partial D}\left|\frac{\partial u}{\partial \nu}\right|^{2}=\int_{\partial D}\left|\frac{\partial u}{\partial \tau}\right|^{2}
$$

from a Fourier series expansion, and so (71) follows.

Inequality (72) follows from (71) and the previous lemma.

For (73) we have that if $\triangle u=0$ and $\left.u\right|_{\partial D} \in H^{1}(\partial D)$, then $\triangle \nabla u=0$ and $\nabla u \in$ $H^{1 / 2}(D)$ by (42). Hence $\left.\nabla u\right|_{\partial D}$ is well-defined, and $\left.\nabla u\right|_{\partial D} \in L^{2}(\partial D)$ from (41). From (44) applied to $\nabla u$,

$$
\|\nabla u\|_{L^{2}\left(\partial D_{h}\right)} \leq c\|\nabla u\|_{L^{2}(\partial D)}
$$

and in particular, using (74),

$$
\left\|\frac{\partial u}{\partial \nu}\right\|_{L^{2}\left(\partial D_{h}\right)} \leq c|u|_{H^{1}(\partial D)}
$$

This establishes (73).

\section{Consistency estimates For the energy}

In this section we compare $E$ and its derivatives at $s$ with $E_{h}$ and its derivatives at $p_{h} s$. Apart from their intrinsic significance, these estimates will be needed in the next section to establish the main convergence results.

Remarks on the Proofs. In the proof of each of the three propositions in this section, Proposition 3.4 is used to estimate the difference between a harmonic function and the corresponding discrete harmonic function, and also to estimate various discrete harmonic "error" terms.

Interpolation results in terms of the $H^{2}$ piecewise seminorm, together with inverse estimates, are used to estimate the $L^{2}$ norm of the quantities

$$
\begin{gathered}
\gamma \circ p_{h} s-I_{h}^{\partial D}\left(\gamma \circ p_{h} s\right), \\
\gamma^{\prime} \circ p_{h} s \xi_{h}-I_{h}^{\partial D}\left(\gamma^{\prime} \circ p_{h} s \xi_{h}\right),
\end{gathered}
$$

and

$$
\gamma^{\prime \prime} \circ p_{h} s \xi_{h}^{2}-I_{h}^{\partial D}\left(\gamma^{\prime \prime} \circ p_{h} s \xi_{h}\right)^{2} .
$$

Since piecewise second derivatives of $p_{h} s$ and $\xi_{h}$ vanish, this enables us to gain an extra power of $h$ over what one might at first expect.

In each of the three proofs the term $I_{2}$ is estimated with an integration by parts. In Proposition 4.1 this improves the order of convergence from $O(h)$ to $O\left(h^{2}\right)$. In the other two propositions, one could not otherwise expect any order of convergence; see also the remark preceding Proposition 4.3 concerning $\left\|\nabla\left(v-v_{h}\right)\right\|_{L^{2}\left(D_{h}\right)}$.

Proposition 4.1. Let $s \in \mathcal{H} \cap C^{2}, \gamma \in C^{2}$. Then

$$
\left|E_{h}\left(p_{h} s\right)-E(s)\right| \leq c h^{2}\|\gamma\|_{C^{2}}^{2}\|s\|_{C^{2}}^{4} .
$$

Proof. Let

$$
u=\Phi(\gamma \circ s), \quad u_{h}=\Phi_{h} I_{h}\left(\gamma \circ p_{h} s\right)
$$


Then

$$
\begin{aligned}
& E_{h}\left(p_{h} s\right)-E(s)=\frac{1}{2} \int_{D_{h}}\left|\nabla u_{h}\right|^{2}-\frac{1}{2} \int_{D}|\nabla u|^{2} \\
& \quad=\frac{1}{2} \int_{D_{h}}\left|\nabla\left(u-u_{h}\right)\right|^{2}-\int_{D_{h}} \nabla u \nabla\left(u-u_{h}\right)-\frac{1}{2} \int_{D \backslash D_{h}}|\nabla u|^{2} \\
& =I_{1}+I_{2}+I_{3} .
\end{aligned}
$$

We will estimate these terms separately.

For $I_{1}$,

$$
\begin{aligned}
\left(2 I_{1}\right)^{1 / 2}= & \left|\Phi(\gamma \circ s)-\Phi_{h} I_{h}\left(\gamma \circ p_{h} s\right)\right|_{H^{1}\left(D_{h}\right)} \\
\leq & \left|\Phi(\gamma \circ s)-\Phi_{h} I_{h}(\gamma \circ s)\right|_{H^{1}\left(D_{h}\right)}+\left|\Phi_{h} I_{h}\left(\gamma \circ s-\gamma \circ p_{h} s\right)\right|_{H^{1}\left(D_{h}\right)} \\
\leq & c h|\gamma \circ s|_{H^{3 / 2}}+\left|\gamma \circ s-\gamma \circ p_{h} s\right|_{H^{1 / 2}}+c h^{1 / 2}\left|\gamma \circ s-\gamma \circ p_{h} s\right|_{H^{1}} \\
& \quad \text { from (64) with } s=3 / 2 \text { and (65) with } s=1 .
\end{aligned}
$$

But

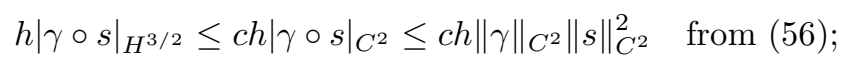

also

$$
\begin{aligned}
\left|\gamma \circ s-\gamma \circ p_{h} s\right|_{H^{1 / 2}} & \leq c\|\gamma\|_{C^{2}}\left(\|s\|_{C^{0,1}}+\left\|p_{h} s\right\|_{C^{0,1}}\right)\left\|s-p_{h} s\right\|_{H^{1 / 2}} \text { by }(61) \\
& \leq c h^{3 / 2}\|\gamma\|_{C^{2}}\|s\|_{C^{1}}\|s\|_{H^{2}} \quad \text { by }(23) \text { and }(22) \\
& \leq c h^{3 / 2}\|\gamma\|_{C^{2}}\|s\|_{C^{2}}^{2}
\end{aligned}
$$

and

$$
\begin{aligned}
h^{1 / 2}\left|\gamma \circ s-\gamma \circ p_{h} s\right|_{H^{1}} & \leq c h^{1 / 2}\|\gamma\|_{C^{2}}\|s\|_{C^{1}}\left\|s-p_{h} s\right\|_{H^{1}} \quad \text { from (62) } \\
& \leq c h^{3 / 2}\|\gamma\|_{C^{2}}\|s\|_{C^{2}}^{2} \quad \text { from (22). }
\end{aligned}
$$

Hence

$$
I_{1} \leq c h^{2}\|\gamma\|_{C^{2}}^{2}\|s\|_{C^{2}}^{4}
$$

For $I_{2}$ one has

$$
\begin{aligned}
\left|I_{2}\right| & =\left|\int_{\partial D_{h}} \frac{\partial u}{\partial \nu}\left(u-u_{h}\right)\right| \\
& \leq\left\|\frac{\partial u}{\partial \nu}\right\|_{L^{2}\left(\partial D_{h}\right)}\left\|u-u_{h}\right\|_{L^{2}\left(\partial D_{h}\right)} \\
& \leq c|u|_{H^{1}(\partial D)}\left\|u-u_{h}\right\|_{L^{2}\left(\partial D_{h}\right)} \quad \text { by }(73) \\
& \leq c\|\gamma\|_{C^{1}}\|s\|_{C^{1}}\left\|u \circ \pi-u_{h} \circ \pi\right\|_{L^{2}(\partial D)} \quad \text { by }(59) \text { and }(14) \\
& \leq c\|\gamma\|_{C^{1}}\|s\|_{C^{1}}\left(\|u \circ \pi-u\|_{L^{2}(\partial D)}+\left\|u-u_{h} \circ \pi\right\|_{L^{2}(\partial D)}\right) .
\end{aligned}
$$

But

$$
\|u \circ \pi-u\|_{L^{2}(\partial D)} \leq c h^{2}|u|_{H^{1}(\partial D)}=c h^{2}|\gamma \circ s|_{H^{1}} \leq c h^{2}\|\gamma\|_{C^{1}}\|s\|_{C^{1}}
$$

by (72) and (59). Also

$$
\begin{aligned}
\left\|u-u_{h} \circ \pi\right\|_{L^{2}(\partial D)} & =\left\|\gamma \circ s-I_{h}^{\partial D}\left(\gamma \circ p_{h} s\right)\right\|_{L^{2}} \quad \text { by }(75) \text { and }(21) \\
& \leq\left\|\gamma \circ s-\gamma \circ p_{h} s\right\|_{L^{2}}+\left\|\gamma \circ p_{h} s-I_{h}^{\partial D}\left(\gamma \circ p_{h} s\right)\right\|_{L^{2}} \\
& \leq\|\gamma\|_{C^{1}}\left\|s-p_{h} s\right\|_{L^{2}}+c h^{2}\left|\gamma \circ p_{h} s\right|_{H^{2}},
\end{aligned}
$$


by (60) and a standard interpolation estimate, where the $H^{2}$ semi-norm is to be understood in a piecewise sense. On each arc segment, $\left(\gamma \circ p_{h} s\right)^{\prime \prime}=\gamma^{\prime \prime} \circ p_{h} s\left(\left(p_{h} s\right)^{\prime}\right)^{2}$ since $\left(p_{h} s\right)^{\prime \prime}=0$, so we can continue the estimate by

$$
\leq c\|\gamma\|_{C^{1}} h^{2}\|s\|_{C^{2}}+c h^{2}\|\gamma\|_{C^{2}}\|s\|_{C^{1}}^{2} \leq c h^{2}\|\gamma\|_{C^{2}}\|s\|_{C^{2}}^{2},
$$

using (24) and (23). Hence

$$
\left|I_{2}\right| \leq c h^{2}\|\gamma\|_{C^{2}}^{2}\|s\|_{C^{2}}^{3}
$$

Finally,

$$
\begin{aligned}
\left|I_{3}\right| & =\frac{1}{2}\|\nabla u\|_{L^{2}\left(D \backslash D_{h}\right)}^{2} \leq c h^{2}|u|_{H^{1}(\partial D)}^{2} \quad \text { by }(71) \\
& =c h^{2}|\gamma \circ s|_{H^{1}}^{2} \leq c h^{2}\|\gamma\|_{C^{1}}^{2}\|s\|_{C^{1}}^{2} .
\end{aligned}
$$

The proposition now follows from (76), (77), (78) and (79).

The next proposition shows that $E_{h}^{\prime}\left(p_{h} s\right)$ is an $O(h)$ approximation to $\left.E^{\prime}(s)\right|_{H_{h}}$ in the $H_{h}^{\prime}$ norm for $\gamma$ and $s$ sufficiently smooth. The actual power of $h$ is important in the proof of Theorem 5.4.

Proposition 4.2. Let $s \in \mathcal{H} \cap C^{2}, \gamma \in C^{3}$. Then for any $\xi_{h} \in H_{h}$

$$
\left|\left\langle E^{\prime}(s), \xi_{h}\right\rangle-\left\langle E_{h}^{\prime}\left(p_{h} s\right), \xi_{h}\right\rangle\right| \leq c h\|\gamma\|_{C^{3}}^{2}\|s\|_{C^{2}}^{3}\left\|\xi_{h}\right\|_{H^{1 / 2}} .
$$

Proof. Define $u$ and $u_{h}$ as in (75), and for $\xi_{h} \in H_{h}$ define

$$
v=\Phi\left(\gamma^{\prime} \circ s \xi_{h}\right), \quad v_{h}=\Phi_{h} I_{h}\left(\gamma^{\prime} \circ p_{h} s \xi_{h}\right) .
$$

Then from (29),

$$
\begin{aligned}
& \left\langle E^{\prime}(s), \xi_{h}\right\rangle-\left\langle E_{h}^{\prime}\left(p_{h} s\right), \xi_{h}\right\rangle \\
& \quad=\int_{D} \nabla u \nabla v-\int_{D_{h}} \nabla u_{h} \nabla v_{h} \\
& =\int_{D_{h}}\left(\nabla u \nabla v-\nabla u_{h} \nabla v_{h}\right)+\int_{D \backslash D_{h}} \nabla u \nabla v \\
& =\int_{D_{h}} \nabla\left(u-u_{h}\right) \nabla v_{h}+\int_{D_{h}} \nabla u \nabla\left(v-v_{h}\right)+\int_{D \backslash D_{h}} \nabla u \nabla v \\
& =I_{1}+I_{2}+I_{3} .
\end{aligned}
$$

Now

$$
\begin{aligned}
\left|I_{1}\right| & \leq\left\|\nabla\left(u-u_{h}\right)\right\|_{L^{2}\left(D_{h}\right)}\left\|\nabla v_{h}\right\|_{L^{2}\left(D_{h}\right)} \\
& \leq c h\|\gamma\|_{C^{2}}\|s\|_{C^{2}}^{2}\left\|\nabla v_{h}\right\|_{L^{2}\left(D_{h}\right)} \quad \text { from }(77) .
\end{aligned}
$$

Also

$$
\begin{aligned}
\left\|\nabla v_{h}\right\|_{L^{2}\left(D_{h}\right)} & =\left|\Phi_{h} I_{h}\left(\gamma^{\prime} \circ p_{h} s \xi_{h}\right)\right|_{H^{1}\left(D_{h}\right)} \\
& \leq\left|\gamma^{\prime} \circ p_{h} s \xi_{h}\right|_{H^{1 / 2}}+c h^{1 / 2}\left|\gamma^{\prime} \circ p_{h} s \xi_{h}\right|_{H^{1}} \quad \text { by }(65) .
\end{aligned}
$$

The first term is estimated by

$$
\begin{aligned}
\left|\gamma^{\prime} \circ p_{h} s \xi_{h}\right|_{H^{1 / 2}} & \leq c\left\|\gamma^{\prime} \circ p_{h} s\right\|_{C^{0,1}}\left\|\xi_{h}\right\|_{H^{1 / 2}} \quad \text { from (49) } \\
& \leq c\|\gamma\|_{C^{2}}\|s\|_{C^{1}}\left\|\xi_{h}\right\|_{H^{1 / 2}} \quad \text { using (23); }
\end{aligned}
$$


the second by

$$
\begin{aligned}
\left|\gamma^{\prime} \circ p_{h} s \xi_{h}\right|_{H^{1}} & \leq c\left\|\gamma^{\prime} \circ p_{h} s\right\|_{C^{0,1}}\left\|\xi_{h}\right\|_{H^{1}} \quad \text { by }(51) \\
& \leq c\|\gamma\|_{C^{2}}\left\|p_{h} s\right\|_{C^{0,1}}\left\|\xi_{h}\right\|_{H^{1}} \quad \text { by }(54) \\
& \leq c h^{-1 / 2}\|\gamma\|_{C^{2}}\|s\|_{C^{1}}\left\|\xi_{h}\right\|_{H^{1 / 2}} \quad \text { by (23) and (19). }
\end{aligned}
$$

So altogether,

$$
\left|I_{1}\right| \leq \operatorname{ch}\|\gamma\|_{C^{2}}^{2}\|s\|_{C^{1}}^{3}\left\|\xi_{h}\right\|_{H^{1 / 2}} .
$$

For $I_{2}$, as for $I_{2}$ in the proof of the previous proposition,

$$
\begin{aligned}
\left|I_{2}\right| & =\left|\int_{\partial D_{h}} \frac{\partial u}{\partial \nu}\left(v-v_{h}\right)\right| \\
& \leq c\|\gamma\|_{C^{1}}\|s\|_{C^{1}}\left(\|v \circ \pi-v\|_{L^{2}(\partial D)}+\left\|v-v_{h} \circ \pi\right\|_{L^{2}(\partial D)}\right) .
\end{aligned}
$$

From (72) and the argument for (82),

$$
\begin{aligned}
\|v \circ \pi-v\|_{L^{2}(\partial D)} & \leq c h^{2}|v|_{H^{1}(\partial D)}=c h^{2}\left|\gamma^{\prime} \circ s \xi_{h}\right|_{H^{1}} \\
& \leq c h^{3 / 2}\|\gamma\|_{C^{2}}\|s\|_{C^{1}}\left\|\xi_{h}\right\|_{H^{1 / 2}} .
\end{aligned}
$$

Also,

$$
\begin{aligned}
\| v- & v_{h} \circ \pi \|_{L^{2}(\partial D)} \\
= & \left\|\gamma^{\prime} \circ s \xi_{h}-I_{h}^{\partial D}\left(\gamma^{\prime} \circ p_{h} s \xi_{h}\right)\right\|_{L^{2}} \\
\leq & \left\|\left(\gamma^{\prime} \circ s-\gamma^{\prime} \circ p_{h} s\right) \xi_{h}\right\|_{L^{2}}+\left\|\gamma^{\prime} \circ p_{h} s \xi_{h}-I_{h}^{\partial D}\left(\gamma^{\prime} \circ p_{h} s \xi_{h}\right)\right\|_{L^{2}} \\
\leq & \|\gamma\|_{C^{2}}\left\|s-p_{h} s\right\|_{C^{0}}\left\|\xi_{h}\right\|_{L^{2}}+c h^{2}\left|\gamma^{\prime} \circ p_{h} s \xi_{h}\right|_{H^{2}} \\
& \quad \text { where the } H^{2} \text { semi-norm is understood in the piecewise sense } \\
\leq \quad & c h^{2}\|\gamma\|_{C^{2}}\|s\|_{C^{2}}\left\|\xi_{h}\right\|_{L^{2}} \quad \text { from }(24) \\
& \quad+c h^{2}\left(\|\gamma\|_{C^{3}}\|s\|_{C^{1}}^{2}\left\|\xi_{h}\right\|_{L^{2}}+\|\gamma\|_{C^{2}}\|s\|_{C^{1}}\left|\xi_{h}\right|_{H^{1}}\right) \quad \text { since on each arc } \\
& \quad \text { segment, }\left(\gamma^{\prime} \circ p_{h} s \xi_{h}\right)^{\prime \prime}=\gamma^{\prime \prime \prime} \circ p_{h} s\left(\left(p_{h} s\right)^{\prime}\right)^{2} \xi_{h}+2 \gamma^{\prime \prime} \circ p_{h} s\left(p_{h} s\right)^{\prime} \xi_{h}^{\prime} \\
\leq & c h^{2}\|\gamma\|_{C^{3}}\|s\|_{C^{2}}^{2}\left\|\xi_{h}\right\|_{H^{1}} \\
\leq & c h^{3 / 2}\|\gamma\|_{C^{3}}\|s\|_{C^{2}}^{2}\left\|\xi_{h}\right\|_{H^{1 / 2}} \quad \text { by }(19) .
\end{aligned}
$$

Altogether,

$$
\left|I_{2}\right| \leq c h^{3 / 2}\|\gamma\|_{C^{3}}^{2}\|s\|_{C^{2}}^{3}\left\|\xi_{h}\right\|_{H^{1 / 2}}
$$

Finally,

$$
\begin{aligned}
\left|I_{3}\right| & \leq\|\nabla u\|_{L^{2}\left(D \backslash D_{h}\right)}\|\nabla v\|_{L^{2}\left(D \backslash D_{h}\right)} \\
& \leq c h\|\gamma\|_{C^{1}}\|s\|_{C^{1}}\|\nabla v\|_{L^{2}\left(D \backslash D_{h}\right)} \quad \text { by }(79) \\
& \leq c h^{2}\|\gamma\|_{C^{1}}\|s\|_{C^{1}}|v|_{H^{1}(\partial D)} \quad \text { by }(71) \\
& \leq c h^{2}\|\gamma\|_{C^{1}}\|s\|_{C^{1}}\left|\gamma^{\prime} \circ s \xi_{h}\right|_{H^{1}} \\
& \leq c h^{2}\|\gamma\|_{C^{1}}\|s\|_{C^{1}}\|\gamma\|_{C^{2}}\|s\|_{C^{1}}\left\|\xi_{h}\right\|_{H^{1}} \quad \text { by }(51) \text { and }(55) \\
& \leq c h^{3 / 2}\|\gamma\|_{C^{2}}^{2}\|s\|_{C^{1}}^{2}\left\|\xi_{h}\right\|_{H^{1 / 2}} \quad \text { by }(19) .
\end{aligned}
$$

The proposition follows from (81), (83), (84) and (85).

Remark. The following proposition, and the related Proposition 5.3, are essential for the proof of the main theorem. Assuming $\gamma$ and $s$ are sufficiently smooth, we see from (87) that if $E^{\prime \prime}(s)$ is positive definite (for example, if $s$ is a strictly stable 
local minimum for $E$ ) then so is $E_{h}^{\prime \prime}\left(p_{h} s\right)$ for $h$ sufficiently small, with the same "ellipticity" constant up to $O\left(h^{1 / 2}|\log h|^{1 / 2}\right)$.

One might hope to estimate the positive term

$$
\left\|\nabla\left(v-v_{h}\right)\right\|_{L^{2}\left(D_{h}\right)}^{2}=\left\|\Phi\left(\gamma^{\prime} \circ s \xi_{h}\right)-\Phi_{h} I_{h}\left(\gamma^{\prime} \circ p_{h} s \xi_{h}\right)\right\|_{H^{1}\left(D_{h}\right)}^{2}
$$

in (87) by $c h^{\alpha}\left\|\xi_{h}\right\|_{H^{1 / 2}}^{2}$ for some $\alpha>0$. But we cannot expect this, as we see by considering the "model" estimates:

$$
\left\|\Phi\left(\xi_{h}\right)-\Phi_{h} I_{h}\left(\xi_{h}\right)\right\|_{H^{1}\left(D_{h}\right)} \leq c h^{1 / 2}\left\|\xi_{h}\right\|_{H^{1}} \leq c\left\|\xi_{h}\right\|_{H^{1 / 2}}
$$

from (64) and (19) - these estimates cannot be improved.

Nonetheless, in Proposition 5.3 we do estimate (86) in case $\xi_{h}=p_{h} \xi$, where $\xi$ is in the negative eigenspace of $E^{\prime \prime}(s)$. The point is that $\xi$ is then smooth by elliptic regularity theory (although $\xi_{h}$ is of course not smooth), and in fact we show in (106) that in this case

$$
\left\|\nabla\left(v-v_{h}\right)\right\|_{L^{2}\left(D_{h}\right)}^{2} \leq c h\left\|\xi_{h}\right\|_{H^{1 / 2}}^{2} .
$$

As a consequence we deduce in Proposition 5.3 that if $E^{\prime \prime}(s)$ is nondegenerate then so is $E_{h}^{\prime \prime}\left(p_{h} s\right)$ for $h$ sufficiently small, with the same nondegeneracy constant up to $O\left(h^{1 / 2}|\log h|^{1 / 2}\right)$.

Proposition 4.3. Let $s \in C^{2} \cap \mathcal{H}$ and $\gamma \in C^{3}$. Then for any $\xi_{h} \in H_{h}$

$$
E_{h}^{\prime \prime}\left(p_{h} s\right)\left(\xi_{h}, \xi_{h}\right)-E^{\prime \prime}(s)\left(\xi_{h}, \xi_{h}\right)=\left\|\nabla\left(v-v_{h}\right)\right\|_{L^{2}\left(D_{h}\right)}^{2}+R
$$

where

$$
v=\Phi\left(\gamma^{\prime} \circ s \xi_{h}\right), \quad v_{h}=\Phi_{h} I_{h}\left(\gamma^{\prime} \circ p_{h} s \xi_{h}\right),
$$

and

$$
|R| \leq \operatorname{ch}^{1 / 2}|\log h|^{1 / 2}\|\gamma\|_{C^{3}}^{2}\|s\|_{C^{2}}^{3}\left\|\xi_{h}\right\|_{H^{1 / 2}}^{2} .
$$

Proof. Let $u$ and $u_{h}$ be as in (75). Let

$$
w=\Phi\left(\gamma^{\prime \prime} \circ s \xi_{h}^{2}\right), \quad w_{h}=\Phi_{h} I_{h}\left(\gamma^{\prime \prime} \circ p_{h} s \xi_{h}^{2}\right) .
$$

From (30),

$$
\begin{aligned}
\left(E^{\prime \prime}(s)-E_{h}^{\prime \prime}\left(p_{h} s\right)\right)\left(\xi_{h}, \xi_{h}\right) \\
=\int_{D}|\nabla v|^{2}+\int_{D} \nabla u \nabla w-\int_{D_{h}}\left|\nabla v_{h}\right|^{2}-\int_{D_{h}} \nabla u_{h} \nabla w_{h} \\
=-\int_{D_{h}}\left|\nabla\left(v-v_{h}\right)\right|^{2}+2 \int_{D_{h}} \nabla v \nabla\left(v-v_{h}\right)+\int_{D_{h}} \nabla\left(u-u_{h}\right) \nabla w_{h} \\
\quad+\int_{D_{h}} \nabla\left(w-w_{h}\right) \nabla u+\int_{D \backslash D_{h}}|\nabla v|^{2}+\int_{D \backslash D_{h}} \nabla u \nabla w \\
=I_{1}+I_{2}+I_{3}+I_{4}+I_{5}+I_{6} .
\end{aligned}
$$

We estimate the terms other than $I_{1}$.

For $I_{2}$ we have

$$
\begin{aligned}
\left|I_{2}\right| & =2\left|\int_{D_{h}} \nabla v \nabla\left(v-v_{h}\right)\right|=2\left|\int_{\partial D_{h}} \frac{\partial v}{\partial \nu}\left(v-v_{h}\right)\right| \\
& \leq 2\left\|\frac{\partial v}{\partial \nu}\right\|_{L^{2}\left(\partial D_{h}\right)}\left\|v-v_{h}\right\|_{L^{2}\left(\partial D_{h}\right)} .
\end{aligned}
$$


The first factor is estimated with the use of (73) and arguing as for (82):

$$
\left\|\frac{\partial v}{\partial \nu}\right\|_{L^{2}\left(\partial D_{h}\right)} \leq c|v|_{H^{1}(\partial D)}=c\left|\gamma^{\prime} \circ s \xi_{h}\right|_{H^{1}} \leq c h^{-1 / 2}\|\gamma\|_{C^{2}}\|s\|_{C^{1}}\left\|\xi_{h}\right\|_{H^{1 / 2}} .
$$

The second factor was estimated in the estimate for $I_{2}$ in the proof of Proposition 4.2:

$$
\left\|v-v_{h}\right\|_{L^{2}\left(\partial D_{h}\right)} \leq c h^{3 / 2}\|\gamma\|_{C^{3}}\|s\|_{C^{2}}^{2}\left\|\xi_{h}\right\|_{H^{1 / 2}} .
$$

Altogether for $I_{2}$,

$$
\left|I_{2}\right| \leq c h\|\gamma\|_{C^{3}}^{2}\|s\|_{C^{2}}^{3}\left\|\xi_{h}\right\|_{H^{1 / 2}}^{2}
$$

For $I_{3}$,

$$
\begin{aligned}
\left|I_{3}\right| & =\left|\int_{D_{h}} \nabla\left(u-u_{h}\right) \nabla w_{h}\right| \leq\left\|\nabla\left(u-u_{h}\right)\right\|_{L^{2}\left(D_{h}\right)}\left\|\nabla w_{h}\right\|_{L^{2}\left(D_{h}\right)} \\
& \leq c h\|\gamma\|_{C^{2}}\|s\|_{C^{2}}^{2}\left\|\nabla w_{h}\right\|_{L^{2}\left(D_{h}\right)} \quad \text { from }(77) .
\end{aligned}
$$

From (65) we get

$$
\begin{aligned}
\left\|\nabla w_{h}\right\|_{L^{2}\left(D_{h}\right)} & =\left|\Phi_{h} I_{h}\left(\gamma^{\prime \prime} \circ p_{h} s \xi_{h}^{2}\right)\right|_{H^{1}\left(D_{h}\right)} \\
& \leq\left|\gamma^{\prime \prime} \circ p_{h} s \xi_{h}^{2}\right|_{H^{1 / 2}}+c h^{1 / 2}\left|\gamma^{\prime \prime} \circ p_{h} s \xi_{h}^{2}\right|_{H^{1}} .
\end{aligned}
$$

But

$$
\begin{aligned}
\left|\gamma^{\prime \prime} \circ p_{h} s \xi_{h}^{2}\right|_{H^{1 / 2}} & \leq c\|\gamma\|_{C^{3}}\|s\|_{C^{1}}\left\|\xi_{h}^{2}\right\|_{H^{1 / 2}} \quad \text { from (49) and (54) } \\
& \leq c\|\gamma\|_{C^{3}}\|s\|_{C^{1}}\left\|\xi_{h}\right\|_{C^{0}}\left\|\xi_{h}\right\|_{H^{1 / 2}} \quad \text { from (48) } \\
& \leq c|\log h|^{1 / 2}\|\gamma\|_{C^{3}}\|s\|_{C^{1}}\left\|\xi_{h}\right\|_{H^{1 / 2}}^{2} \quad \text { from (20). }
\end{aligned}
$$

Similarly,

$$
\begin{aligned}
\left|\gamma^{\prime \prime} \circ p_{h} s \xi_{h}^{2}\right|_{H^{1}} & \leq c\|\gamma\|_{C^{3}}\|s\|_{C^{1}}\left\|\xi_{h}^{2}\right\|_{H^{1}} \quad \text { using (51) } \\
& \leq c\|\gamma\|_{C^{3}}\|s\|_{C^{1}}\left\|\xi_{h}\right\|_{C^{0}}\left\|\xi_{h}\right\|_{H^{1}} \quad \text { from (50) } \\
& \leq c h^{-1 / 2}|\log h|^{1 / 2}\|\gamma\|_{C^{3}}\|s\|_{C^{1}}\left\|\xi_{h}\right\|_{H^{1 / 2}}^{2}
\end{aligned}
$$

from (20) and (19). Hence

$$
\left\|\nabla w_{h}\right\|_{L^{2}\left(D_{h}\right)} \leq c|\log h|^{1 / 2}\|\gamma\|_{C^{3}}\|s\|_{C^{1}}\left\|\xi_{h}\right\|_{H^{1 / 2}}^{2},
$$

and so

$$
\left|I_{3}\right| \leq c h|\log h|^{1 / 2}\|\gamma\|_{C^{3}}^{2}\|s\|_{C^{2}}^{3}\left\|\xi_{h}\right\|_{H^{1 / 2}}^{2} .
$$

For $I_{4}$ we have

$$
I_{4}=\int_{D_{h}} \nabla\left(w-w_{h}\right) \nabla u=\int_{D_{h}}\left(w-w_{h}\right) \frac{\partial u}{\partial \nu},
$$

and so, as for $I_{2}$ in the proof of Proposition 4.1,

$$
\left|I_{4}\right| \leq c\|\gamma\|_{C^{1}}\|s\|_{C^{1}}\left(\|w \circ \pi-w\|_{L^{2}(\partial D)}+\left\|w-w_{h} \circ \pi\right\|_{L^{2}(\partial D)}\right) .
$$

But

$$
\begin{aligned}
\|w \circ \pi-w\|_{L^{2}(\partial D)} & \leq c h^{2}|w|_{H^{1}(\partial D)} \quad \text { by }(72) \\
& \leq c h^{2}\left|\gamma^{\prime \prime} \circ s \xi_{h}^{2}\right|_{H^{1}} \\
& \leq c h^{3 / 2}|\log h|^{1 / 2}\|\gamma\|_{C^{3}}\|s\|_{C^{1}}\left\|\xi_{h}\right\|_{H^{1 / 2}}^{2} \quad \text { as for (90). }
\end{aligned}
$$


Also

$$
\begin{aligned}
& \left\|w-w_{h} \circ \pi\right\|_{L^{2}(\partial D)} \\
& =\left\|\gamma^{\prime \prime} \circ s \xi_{h}^{2}-I_{h}^{\partial D}\left(\gamma^{\prime \prime} \circ p_{h} s \xi_{h}^{2}\right)\right\|_{L^{2}} \quad \text { from }(88) \text { and }(21) \\
& \leq\left\|\gamma^{\prime \prime} \circ s \xi_{h}^{2}-\gamma^{\prime \prime} \circ p_{h} s \xi_{h}^{2}\right\|_{L^{2}}+\left\|\gamma^{\prime \prime} \circ p_{h} s \xi_{h}^{2}-I_{h}^{\partial D}\left(\gamma^{\prime \prime} \circ p_{h} s \xi_{h}^{2}\right)\right\|_{L^{2}} \\
& \leq\left\|\gamma^{\prime \prime} \circ s-\gamma^{\prime \prime} \circ p_{h} s\right\|_{C^{0}}\left\|\xi_{h}\right\|_{L^{4}}^{2}+c h\left|\gamma^{\prime \prime} \circ p_{h} s \xi_{h}^{2}\right|_{H^{1}} \\
& \leq \quad c\|\gamma\|_{C^{3}}\left\|s-p_{h} s\right\|_{C^{0}}\left\|\xi_{h}\right\|_{H^{1 / 2}}^{2}+c h^{1 / 2}|\log h|^{1 / 2}\|\gamma\|_{C^{3}}\|s\|_{C^{1}}\left\|\xi_{h}\right\|_{H^{1 / 2}}^{2} \\
& \quad \text { using a Sobolev embedding theorem and }(90) \\
& \leq \quad c h^{1 / 2}|\log h|^{1 / 2}\|\gamma\|_{C^{3}}\|s\|_{C^{1}}\left\|\xi_{h}\right\|_{H^{1 / 2}}^{2} \quad \text { from }(24) .
\end{aligned}
$$

Hence

$$
\left|I_{4}\right| \leq c h^{1 / 2}|\log h|^{1 / 2}\|\gamma\|_{C^{3}}^{2}\|s\|_{C^{1}}^{2}\left\|\xi_{h}\right\|_{H^{1 / 2}}^{2} .
$$

From the final part of the argument for $I_{3}$ in the proof of the previous proposition,

$$
I_{5}=\|\nabla v\|_{L^{2}\left(D \backslash D_{h}\right)}^{2} \leq c h\|\gamma\|_{C^{2}}^{2}\|s\|_{C^{1}}^{2}\left\|\xi_{h}\right\|_{H^{1 / 2}}^{2} .
$$

Finally,

$$
\begin{aligned}
\left|I_{6}\right| & \leq\|\nabla u\|_{L^{2}\left(D \backslash D_{h}\right)}\|\nabla w\|_{L^{2}\left(D \backslash D_{h}\right)} \\
& \leq c h^{2}|u|_{H^{1}(\partial D)}|w|_{H^{1}(\partial D)} \quad \text { by }(71) \\
& =c h^{2}|\gamma \circ s|_{H^{1}}\left|\gamma^{\prime \prime} \circ s \xi_{h}^{2}\right|_{H^{1}} \\
& \leq c h^{2}\|\gamma\|_{C^{1}}\|s\|_{C^{1}}\left|\gamma^{\prime \prime} \circ s \xi_{h}^{2}\right|_{H^{1}} \quad \text { by (59) } \\
& \leq c h^{3 / 2}|\log h|^{1 / 2}\|\gamma\|_{C^{3}}^{2}\|s\|_{C^{1}}^{2}\left\|\xi_{h}\right\|_{H^{1 / 2}}^{2} \quad \text { as for }(90) .
\end{aligned}
$$

Now we collect the estimates (89), (91), (93), (94) and (95), and prove the proposition.

\section{The MAin ERRor estimates}

We will apply the following quantitative version of the Inverse Function Theorem with $\mathcal{X}=\mathcal{H}_{h}$ and $Y=H_{h}^{\prime}$ in the proof of Theorem 5.4.

Lemma 5.1. Let $\mathcal{X}$ be an affine Banach space with Banach space $X$ as tangent space, and let $Y$ be a Banach space. Suppose $x_{0} \in \mathcal{X}$ and $f \in C^{1}(\mathcal{X}, Y)$. Assume there are positive constants $\alpha, \beta, \delta$ and $\epsilon$ such that

$$
\begin{aligned}
\left\|f\left(x_{0}\right)\right\|_{Y} & \leq \delta \\
\left\|f^{\prime}\left(x_{0}\right)^{-1}\right\|_{L(Y, X)} & \leq \alpha^{-1}, \\
\left\|f^{\prime}(x)-f^{\prime}\left(x_{0}\right)\right\|_{L(X, Y)} & \leq \beta \text { for all } x \in \bar{B}_{\epsilon}\left(x_{0}\right),
\end{aligned}
$$

where

$$
\beta<\alpha, \quad \delta \leq(\alpha-\beta) \epsilon .
$$

Then there exists a unique $x_{*} \in \bar{B}_{\epsilon}\left(x_{0}\right)$ such that $f\left(x_{*}\right)=0$.

Proof. This follows from the proof of the Inverse Function Theorem in [Be, pp. 113-114]. The modifications necessary since $\mathcal{X}$ is an affine space are trivial.

The next proposition establishes that for any $\beta>0$,

$$
\left\|E_{h}^{\prime \prime}\left(p_{h} s\right)-E_{h}^{\prime \prime}\left(p_{h} s+\eta_{h}\right)\right\|_{\left(H_{h} \times H_{h}\right)^{\prime}} \leq \beta
$$


provided $|\log h|\left\|\eta_{h}\right\|_{H^{1 / 2}}$ is sufficiently small (depending on $\beta$ but independent of $h$ ). This will be used to establish the appropriate version of (98) in the proof of Proposition 5.4.

Remark on the Proof. The precise form of estimate (61), and the inverse estimate (20), are used to control $\left|g \circ s_{h}-g \circ\left(s_{h}+\eta_{h}\right)\right|_{H^{1 / 2}}$ for $g=\gamma, \gamma^{\prime}$ and $\gamma^{\prime \prime}$.

Proposition 5.2. Let $s \in \mathcal{H} \cap C^{1}$ and $\gamma \in C^{4}$. Then for any $\eta_{h}, \xi_{h} \in H_{h}$

$$
\begin{aligned}
& \left|\left(E_{h}^{\prime \prime}\left(p_{h} s\right)-E_{h}^{\prime \prime}\left(p_{h} s+\eta_{h}\right)\right)\left(\xi_{h}, \xi_{h}\right)\right| \\
& \quad \leq c\left(1+|\log h|^{1 / 2}\left\|\eta_{h}\right\|_{H^{1 / 2}}\right)^{2}|\log h|\left\|\eta_{h}\right\|_{H^{1 / 2}}\|\gamma\|_{C^{4}}^{2}\|s\|_{C^{1}}^{2}\left\|\xi_{h}\right\|_{H^{1 / 2}}^{2} .
\end{aligned}
$$

Proof. For simplicity we write $s_{h}$ for $p_{h} s$.

From (30),

$$
\begin{aligned}
& \left(E_{h}^{\prime \prime}\left(s_{h}\right)-E_{h}^{\prime \prime}\left(s_{h}+\eta_{h}\right)\right)\left(\xi_{h}, \xi_{h}\right) \\
& =\int_{D_{h}}\left|\nabla v_{h}\right|^{2}+\int_{D_{h}} \nabla u_{h} \nabla w_{h}-\int_{D_{h}}\left|\nabla \bar{v}_{h}\right|^{2}-\int_{D_{h}} \nabla \bar{u}_{h} \nabla \bar{w}_{h}
\end{aligned}
$$

with

$$
\begin{array}{lll}
u_{h}=\Phi_{h} I_{h}\left(\gamma \circ s_{h}\right), & \bar{u}_{h}=\Phi_{h} I_{h}\left(\gamma \circ\left(s_{h}+\eta_{h}\right)\right), \\
v_{h}=\Phi_{h} I_{h}\left(\gamma^{\prime} \circ s_{h} \xi_{h}\right), & \bar{v}_{h}=\Phi_{h} I_{h}\left(\gamma^{\prime} \circ\left(s_{h}+\eta_{h}\right) \xi_{h}\right), \\
w_{h}=\Phi_{h} I_{h}\left(\gamma^{\prime \prime} \circ s_{h} \xi_{h}^{2}\right), & \bar{w}_{h}=\Phi_{h} I_{h}\left(\gamma^{\prime \prime} \circ\left(s_{h}+\eta_{h}\right) \xi_{h}^{2}\right) .
\end{array}
$$

So

$$
\begin{aligned}
& \left|\left(E_{h}^{\prime \prime}\left(s_{h}\right)-E_{h}^{\prime \prime}\left(s_{h}+\eta_{h}\right)\right)\left(\xi_{h}, \xi_{h}\right)\right| \\
& \leq \int_{D_{h}}\left|\nabla\left(v_{h}-\bar{v}_{h}\right)\right|\left(\left|\nabla v_{h}\right|+\left|\nabla \bar{v}_{h}\right|\right)+\int_{D_{h}}\left|\nabla\left(u_{h}-\bar{u}_{h}\right)\right|\left|\nabla w_{h}\right| \\
& +\int_{D_{h}}\left|\nabla \bar{u}_{h}\right|\left|\nabla\left(w_{h}-\bar{w}_{h}\right)\right| \\
& \leq\left\|\nabla\left(u_{h}-\bar{u}_{h}\right)\right\|_{L^{2}\left(D_{h}\right)}\left\|\nabla w_{h}\right\|_{L^{2}\left(D_{h}\right)} \\
& +\left\|\nabla\left(v_{h}-\bar{v}_{h}\right)\right\|_{L^{2}\left(D_{h}\right)}\left(\left\|\nabla v_{h}\right\|_{L^{2}\left(D_{h}\right)}+\left\|\nabla \bar{v}_{h}\right\|_{L^{2}\left(D_{h}\right)}\right) \\
& +\left\|\nabla\left(w_{h}-\bar{w}_{h}\right)\right\|_{L^{2}\left(D_{h}\right)}\left\|\nabla \bar{u}_{h}\right\|_{L^{2}\left(D_{h}\right)} \\
& =A_{1} B_{1}+A_{2}\left(B_{21}+B_{22}\right)+A_{3} B_{3} \text {. }
\end{aligned}
$$

Now we estimate these terms separately.

By (65)

$$
\begin{aligned}
A_{1}= & \left|\Phi_{h} I_{h}\left(\gamma \circ s_{h}-\gamma \circ\left(s_{h}+\eta_{h}\right)\right)\right|_{H^{1}\left(D_{h}\right)} \\
\leq & \left|\gamma \circ s_{h}-\gamma \circ\left(s_{h}+\eta_{h}\right)\right|_{H^{1 / 2}}+c h^{1 / 2}\left|\gamma \circ s_{h}-\gamma \circ\left(s_{h}+\eta_{h}\right)\right|_{H^{1}} \\
\leq & c\|\gamma\|_{C^{2}}\left(\left\|s_{h}\right\|_{C^{0,1}}+\left\|\eta_{h}\right\|_{C^{0}}\right)\left\|\eta_{h}\right\|_{H^{1 / 2}}+c h^{1 / 2}\|\gamma\| C_{C^{2}}\left\|s_{h}\right\|_{C^{0,1}}\left\|\eta_{h}\right\|_{H^{1}} \\
& \quad \text { by }(61) \text { and }(62) \\
\leq & c\|\gamma\|_{C^{2}}\|s\|_{C^{1}}\left\|\eta_{h}\right\|_{H^{1 / 2}}\left(1+|\log h|^{1 / 2}\left\|\eta_{h}\right\|_{H^{1 / 2}}\right) \\
& +c\|\gamma\|_{C^{2}}\|s\|_{C^{1}}\left\|\eta_{h}\right\|_{H^{1 / 2}} \text { by }(20),(19) \text { and }(23) \\
\leq & c\left(1+|\log h|^{1 / 2}\left\|\eta_{h}\right\|_{H^{1 / 2}}\right)\left\|\eta_{h}\right\|_{H^{1 / 2}}\|\gamma\|_{C^{2}}\|s\|_{C^{1}} .
\end{aligned}
$$


Again from (65)

$$
\begin{aligned}
A_{2}= & \left|\Phi_{h} I_{h}\left(\gamma^{\prime} \circ s_{h} \xi_{h}-\gamma^{\prime} \circ\left(s_{h}+\eta_{h}\right) \xi_{h}\right)\right|_{H^{1}\left(D_{h}\right)} \\
\leq & \left|\left(\gamma^{\prime} \circ s_{h}-\gamma^{\prime} \circ\left(s_{h}+\eta_{h}\right)\right) \xi_{h}\right|_{H^{1 / 2}} \\
& +c h^{1 / 2}\left|\left(\gamma^{\prime} \circ s_{h}-\gamma^{\prime} \circ\left(s_{h}+\eta_{h}\right)\right) \xi_{h}\right|_{H^{1}} \\
\leq & \left|\gamma^{\prime} \circ s_{h}-\gamma^{\prime} \circ\left(s_{h}+\eta_{h}\right)\right|_{H^{1 / 2}}\left\|\xi_{h}\right\|_{C^{0}} \\
& +\left\|\gamma^{\prime} \circ s_{h}-\gamma^{\prime} \circ\left(s_{h}+\eta_{h}\right)\right\|_{C^{0}}\left|\xi_{h}\right|_{H^{1 / 2}} \quad \text { by }(48) \\
& +c h^{1 / 2}\left\|\gamma^{\prime} \circ s_{h}-\gamma^{\prime} \circ\left(s_{h}+\eta_{h}\right)\right\|_{C^{0}}\left|\xi_{h}\right|_{H^{1}} \\
& +c h^{1 / 2}\left|\gamma^{\prime} \circ s_{h}-\gamma^{\prime} \circ\left(s_{h}+\eta_{h}\right)\right|_{H^{1}}\left\|\xi_{h}\right\|_{C^{0}} \quad \text { by }(50) \\
\leq \quad & c\left(1+|\log h|^{1 / 2}\left\|\eta_{h}\right\|_{H^{1 / 2}}\right)\left\|\eta_{h}\right\|_{H^{1 / 2}}\|\gamma\|_{C^{3}}\|s\|_{C^{1}}\left\|\xi_{h}\right\|_{C^{0}} \\
& +c\|\gamma\|_{C^{2}}\left\|\eta_{h}\right\|_{C^{0}}\left|\xi_{h}\right|_{H^{1 / 2}}+c h^{1 / 2}\|\gamma\|_{C^{2}}\left\|\eta_{h}\right\|_{C^{0}}\left|\xi_{h}\right|_{H^{1}} \quad \text { using the argu- } \\
& \text { ment for } A_{1} \text { on the first and last terms in the preceding inequality } \\
\leq & c\left(1+|\log h|^{1 / 2}\left\|\eta_{h}\right\|_{H^{1 / 2}}\right)|\log h|^{1 / 2}\left\|\eta_{h}\right\|_{H^{1 / 2}}\|\gamma\|_{C^{3}}\|s\|_{C^{1}}\left\|\xi_{h}\right\|_{H^{1 / 2}},
\end{aligned}
$$

again using the inverse estimates (20) and (19) on $\eta_{h}$ and $\xi_{h}$.

In the same way we proceed for $A_{3}$ to first obtain the analogue of the next-to-last inequality above:

$$
\begin{aligned}
A_{3}= & \left|\Phi_{h} I_{h}\left(\gamma^{\prime \prime} \circ s_{h} \xi_{h}^{2}-\gamma^{\prime \prime} \circ\left(s_{h}+\eta_{h}\right) \xi_{h}^{2}\right)\right|_{H^{1}\left(D_{h}\right)} \\
\leq & c\left(1+|\log h|^{1 / 2}\left\|\eta_{h}\right\|_{H^{1 / 2}}\right)\left\|\eta_{h}\right\|^{1 / 2}\|\gamma\|_{C^{4}}\|s\|_{C^{1}}\left\|\xi_{h}^{2}\right\|_{C^{0}} \\
& +c\|\gamma\|_{C^{3}}\left\|\eta_{h}\right\|_{C^{0}}\left|\xi_{h}^{2}\right|_{H^{1 / 2}}+c h^{1 / 2}\|\gamma\|_{C^{3}}\left\|\eta_{h}\right\|_{C^{0}}\left|\xi_{h}^{2}\right|_{H^{1}} \\
\leq & c\left(1+|\log h|^{1 / 2}\left\|\eta_{h}\right\|_{H^{1 / 2}}\right)|\log h|\left\|\eta_{h}\right\|_{H^{1 / 2}}\|\gamma\|_{C^{4}}\|s\|_{C^{1}}\left\|\xi_{h}\right\|_{H^{1 / 2}}^{2},
\end{aligned}
$$

from the inverse estimates (19) and (20) applied to $\eta_{h}$ and $\xi_{h}$, and since

$$
\left\|\xi_{h}^{2}\right\|_{H^{1 / 2}} \leq 2\left\|\xi_{h}\right\|_{C^{0}}\left\|\xi_{h}\right\|_{H^{1 / 2}}, \quad\left\|\xi_{h}^{2}\right\|_{H^{1}} \leq 2\left\|\xi_{h}\right\|_{C^{0}}\left\|\xi_{h}\right\|_{H^{1}} .
$$

For $B_{3}$,

$$
\begin{aligned}
B_{3} & =\left|\Phi_{h} I_{h}\left(\gamma \circ\left(s_{h}+\eta_{h}\right)\right)\right|_{H^{1}\left(D_{h}\right)} \\
& \leq\left|\gamma \circ\left(s_{h}+\eta_{h}\right)\right|_{H^{1 / 2}}+c h^{1 / 2}\left|\gamma \circ\left(s_{h}+\eta_{h}\right)\right|_{H^{1}} \quad \text { by }(65) \\
& \leq\|\gamma\|_{C^{1}}\left(\left\|s_{h}\right\|_{H^{1 / 2}}+\left\|\eta_{h}\right\|_{H^{1 / 2}}\right)+c h^{1 / 2}\|\gamma\|_{C^{1}}\left(\left\|s_{h}\right\|_{H^{1}}+\left\|\eta_{h}\right\|_{H^{1}}\right) \\
& \leq c\|\gamma\|_{C^{1}}\left(\left\|s_{h}\right\|_{H^{1 / 2}}+\left\|\eta_{h}\right\|_{H^{1 / 2}}\right) \quad \text { by }(19) \\
& \leq c\left(1+\left\|\eta_{h}\right\|_{H^{1 / 2}}\right)\|\gamma\|_{C^{1}}\|s\|_{C^{1}} .
\end{aligned}
$$


For $B_{22}$,

$$
\begin{aligned}
B_{22}= & \left|\Phi_{h} I_{h}\left(\gamma^{\prime} \circ\left(s_{h}+\eta_{h}\right) \xi_{h}\right)\right|_{H^{1}\left(D_{h}\right)} \\
\leq & \left|\gamma^{\prime} \circ\left(s_{h}+\eta_{h}\right) \xi_{h}\right|_{H^{1 / 2}}+c h^{1 / 2}\left|\gamma^{\prime} \circ\left(s_{h}+\eta_{h}\right) \xi_{h}\right|_{H^{1}} \quad \text { by }(65) \\
\leq & \left\|\gamma^{\prime} \circ\left(s_{h}+\eta_{h}\right)\right\|_{C^{0}}\left|\xi_{h}\right|_{H^{1 / 2}}+\left|\gamma^{\prime} \circ\left(s_{h}+\eta_{h}\right)\right|_{H^{1 / 2}}\left\|\xi_{h}\right\|_{C^{0}} \\
& +c h^{1 / 2}\left(\left\|\gamma^{\prime} \circ\left(s_{h}+\eta_{h}\right)\right\|_{C^{0}}\left|\xi_{h}\right|_{H^{1}}+\left|\gamma^{\prime} \circ\left(s_{h}+\eta_{h}\right)\right|_{H^{1}}\left\|\xi_{h}\right\|_{C^{0}}\right) \\
& \operatorname{by}(48) \\
\leq & c\|\gamma\|_{C^{1}}\left|\xi_{h}\right|_{H^{1 / 2}}+c\|\gamma\|_{C^{2}}\left|s_{h}+\eta_{h}\right|_{H^{1 / 2}}\left\|\xi_{h}\right\|_{C^{0}} \\
& +c h^{1 / 2}\left(\|\gamma\|_{C^{1}}\left|\xi_{h}\right|_{H^{1}}+\|\gamma\|_{C^{2}}\left\|s_{h}+\eta_{h}\right\|_{H^{1}}\left\|\xi_{h}\right\|_{C^{0}}\right) \\
\leq & c\|\gamma\|_{C^{2}}\left(\left\|s_{h}+\eta_{h}\right\|_{H^{1 / 2}}\right)|\log h|^{1 / 2}\left\|\xi_{h}\right\|_{H^{1 / 2}} \quad \text { by }(19) \text { and }(20) \\
\leq & c\left(1+\left\|\eta_{h}\right\|_{H^{1 / 2}}\right)|\log h|^{1 / 2}\|\gamma\|_{C^{2}}\|s\|_{C^{1}}\left\|\xi_{h}\right\|_{H^{1 / 2}} .
\end{aligned}
$$

For $B_{21}$ we have the same estimate with $\eta_{h}$ set equal to 0 :

$$
B_{21} \leq c|\log h|^{1 / 2}\|\gamma\|_{C^{2}}\|s\|_{C^{1}}\left\|\xi_{h}\right\|_{H^{1 / 2}} .
$$

Finally,

$$
\begin{aligned}
B_{1}= & \left|\Phi_{h} I_{h}\left(\gamma^{\prime \prime} \circ s_{h} \xi_{h}^{2}\right)\right|_{H^{1}\left(D_{h}\right)} \\
\leq & \left|\gamma^{\prime \prime} \circ s_{h} \xi_{h}^{2}\right|_{H^{1 / 2}}+c h^{1 / 2}\left|\gamma^{\prime \prime} \circ s_{h} \xi_{h}^{2}\right|_{H^{1}} \quad \text { by }(65) \\
\leq & \left\|\gamma^{\prime \prime} \circ s_{h}\right\|_{C^{0}}\left|\xi_{h}^{2}\right|_{H^{1 / 2}}+\left|\gamma^{\prime \prime} \circ s_{h}\right|_{H^{1 / 2}}\left\|\xi_{h}^{2}\right\|_{C^{0}} \\
& +c h^{1 / 2}\left(\left\|\gamma^{\prime \prime} \circ s_{h}\right\|_{C^{0}}\left|\xi_{h}^{2}\right|_{H^{1}}+\left|\gamma^{\prime \prime} \circ s_{h}\right|_{H^{1}}\left\|\xi_{h}^{2}\right\|_{C^{0}}\right) \\
\leq & c\|\gamma\|_{C^{2}}\left\|\xi_{h}\right\|_{C^{0}}\left\|\xi_{h}\right\|_{H^{1 / 2}}+c\|\gamma\|_{C^{3}}\left\|s_{h}\right\|_{H^{1 / 2}}\left\|\xi_{h}\right\|_{C^{0}}^{2} \\
& +c h^{1 / 2}\left(\|\gamma\|_{C^{2}}\left\|\xi_{h}\right\|_{C^{0}}\left\|\xi_{h}\right\|_{H^{1}}+\|\gamma\|_{C^{3}}\left\|s_{h}\right\|_{H^{1}}\left\|\xi_{h}\right\|_{C^{0}}^{2}\right) \\
\leq & c \mid \log h\|\gamma\|_{C^{3}}\|s\|_{C^{1}}\left\|\xi_{h}\right\|_{H^{1 / 2}}^{2} .
\end{aligned}
$$

Substituting the previous estimates in (100), we get

$$
\begin{aligned}
& \left|\left(E_{h}^{\prime \prime}\left(s_{h}\right)-E_{h}^{\prime \prime}\left(s_{h}+\eta_{h}\right)\right)\left(\xi_{h}, \xi_{h}\right)\right| \\
& \leq c\left(1+|\log h|^{1 / 2}\left\|\eta_{h}\right\|_{H^{1 / 2}}\right)\left\|\eta_{h}\right\|_{H^{1 / 2}}\|\gamma\|_{C^{2}}\|s\|_{C^{1}} \\
& \quad \times|\log h|\|\gamma\|_{C^{3}}\|s\|_{C^{1}}\left\|\xi_{h}\right\|_{H^{1 / 2}}^{2} \\
& +c\left(1+|\log h|^{1 / 2}\left\|\eta_{h}\right\|_{H^{1 / 2}}\right)|\log h|^{1 / 2}\left\|\eta_{h}\right\|_{H^{1 / 2}}\|\gamma\|_{C^{3}}\|s\|_{C^{1}}\left\|\xi_{h}\right\|_{H^{1 / 2}} \\
& \quad \times\left(1+\left\|\eta_{h}\right\|_{H^{1 / 2}}\right)|\log h|^{1 / 2}\|\gamma\|_{C^{2}}\|s\|_{C^{1}}\left\|\xi_{h}\right\|_{H^{1 / 2}} \\
& +c\left(1+\left\|\eta_{h}\right\|_{H^{1 / 2}}\right)\|\gamma\|_{C^{1}}\|s\|_{C^{1}} \\
& \quad \times\left(1+|\log h|^{1 / 2}\left\|\eta_{h}\right\|_{H^{1 / 2}}\right)|\log h|\left\|\eta_{h}\right\|_{H^{1 / 2}}\|\gamma\|_{C^{4}}\|s\|_{C^{1}}\left\|\xi_{h}\right\|_{H^{1 / 2}}^{2} \\
& \leq c\left(1+|\log h|^{1 / 2}\left\|\eta_{h}\right\|_{H^{1 / 2}}\right)^{2}|\log h|\left\|\eta_{h}\right\|_{H^{1 / 2}}\|\gamma\|_{C^{4}}^{2}\|s\|_{C^{1}}^{2}\left\|\xi_{h}\right\|_{H^{1 / 2}}^{2} .
\end{aligned}
$$

It follows from the next result that $E_{h}^{\prime \prime}\left(p_{h} s\right)$ is nondegenerate with nondegeneracy constant arbitrarily close to $\lambda$, provided $h$ is sufficiently small. This will be used to establish the appropriate version of (97) in the proof of Theorem 5.4. 
Remark on the Proof. (See also the Remark preceding Proposition 4.3). Nondegeneracy for "interpolants" of functions from the positive and negative spaces for $E^{\prime \prime}(s)$ must be treated separately - see the separate treatment of $I_{1}$ and $I_{2}$ below. In the first case, nondegeneracy follows more or less directly from Proposition 4.3. In the second case, the term $\left\|\nabla\left(v-v_{h}\right)\right\|_{L^{2}\left(D_{h}\right)}^{2}$, where $v=\Phi\left(\gamma^{\prime} \circ s \xi_{h}^{(-)}\right)$and $v_{h}=\Phi_{h} I_{h}\left(\gamma^{\prime} \circ s_{h} \xi_{h}^{(-)}\right)$, must be estimated. The main points are to apply the regularity results (34) and Proposition 2.2 to members of the negative space.

Proposition 5.3. Let $s \in \mathcal{H} \cap C^{2}$ and $\gamma \in C^{3}$. Suppose $E^{\prime \prime}(s)$ is nondegenerate with nondegeneracy constant $\lambda$, as in (13). Let $\nu$ be as in (12). Then

$$
E_{h}^{\prime \prime}\left(p_{h} s\right)\left(\xi_{h}, \xi_{h}^{(+)}-\xi_{h}^{(-)}\right) \geq\left(\lambda-c h^{1 / 2}|\log h|^{1 / 2}\|\gamma\|_{C^{3}}^{2} \nu^{2}\|s\|_{C^{2}}^{4}\right)\left\|\xi_{h}\right\|_{H^{1 / 2}}^{2}
$$

for every $\xi_{h} \in H_{h}$.

Proof. We again denote $p_{h} s$ by $s_{h}$ and split

$$
\begin{aligned}
E_{h}^{\prime \prime}\left(s_{h}\right)\left(\xi_{h}, \xi_{h}^{(+)}-\xi_{h}^{(-)}\right) & =E_{h}^{\prime \prime}\left(s_{h}\right)\left(\xi_{h}^{(+)}, \xi_{h}^{(+)}\right)-E_{h}^{\prime \prime}\left(s_{h}\right)\left(\xi_{h}^{(-)}, \xi_{h}^{(-)}\right) \\
& =I_{1}+I_{2} .
\end{aligned}
$$

It follows from (87), after discarding a positive term, that

$$
\begin{aligned}
I_{1} \geq & E^{\prime \prime}(s)\left(\xi_{h}^{(+)}, \xi_{h}^{(+)}\right)-c h^{1 / 2}|\log h|^{1 / 2}\|\gamma\|_{C^{3}}^{2}\|s\|_{C^{2}}^{3}\left\|\xi_{h}^{(+)}\right\|_{H^{1 / 2}}^{2} \\
= & E^{\prime \prime}(s)\left(\xi_{h}^{+}, \xi_{h}^{+}\right)+E^{\prime \prime}(s)\left(\left(\xi_{h}^{(+)}, \xi_{h}^{(+)}\right)-\left(\xi_{h}^{+}, \xi_{h}^{+}\right)\right) \\
& -c h^{1 / 2}|\log h|^{1 / 2}\|\gamma\|_{C^{3}}^{2}\|s\|_{C^{2}}^{3}\left\|\xi_{h}^{(+)}\right\|_{H^{1 / 2}}^{2} .
\end{aligned}
$$

Using the notation of (13), there exists $\lambda^{+}>0$ such that

$$
E^{\prime \prime}(s)\left(\xi_{h}^{+}, \xi_{h}^{+}\right) \geq \lambda^{+}\left\|\xi_{h}^{+}\right\|_{H^{1 / 2}}^{2} .
$$

Also

$$
\begin{aligned}
& \left|E^{\prime \prime}(s)\left(\left(\xi_{h}^{(+)}, \xi_{h}^{(+)}\right)-\left(\xi_{h}^{+}, \xi_{h}^{+}\right)\right)\right| \\
& \quad=E^{\prime \prime}(s)\left(\xi_{h}^{(+)}-\xi_{h}^{+}, \xi_{h}^{(+)}+\xi_{h}^{+}\right) \\
& \quad \leq c\|\gamma\|_{C^{2}}^{2}\|s\|_{C^{1}}^{2}\left\|\xi_{h}^{(+)}-\xi_{h}^{+}\right\|_{H^{1 / 2}}\left\|\xi_{h}^{(+)}+\xi_{h}^{+}\right\|_{H^{1 / 2}} \quad \text { by }(10) \\
& \quad \leq c h\|\gamma\|_{C^{2}}^{2} \nu^{2}\|s\|_{C^{1}}^{2}\left\|\xi_{h}\right\|_{H^{1 / 2}}^{2} \text { by }(36) .
\end{aligned}
$$

Using (36) again, $I_{1}$ can then be estimated from below by

$$
I_{1} \geq \lambda^{+}\left\|\xi_{h}^{+}\right\|_{H^{1 / 2}}^{2}-c h^{1 / 2}|\log h|^{1 / 2}\|\gamma\|_{C^{3}}^{2} \nu^{2}\|s\|_{C^{2}}^{3}\left\|\xi_{h}\right\|_{H^{1 / 2}}^{2} .
$$

With similar arguments, but now keeping all terms in (87) and using the notation of (13), we get

$$
\begin{aligned}
I_{2} \geq & \lambda^{-}\left\|\xi_{h}^{-}\right\|_{H^{1 / 2}}^{2}-c h^{1 / 2}|\log h|^{1 / 2}\|\gamma\|_{C^{3}}^{2} \nu^{2}\|s\|_{C^{2}}^{3}\left\|\xi_{h}\right\|_{H^{1 / 2}}^{2} \\
& -\left\|\nabla\left(v-v_{h}\right)\right\|_{L^{2}\left(D_{h}\right)}^{2},
\end{aligned}
$$

where, here,

$$
v=\Phi\left(\gamma^{\prime} \circ s \xi_{h}^{(-)}\right), \quad v_{h}=\Phi_{h} I_{h}\left(\gamma^{\prime} \circ s_{h} \xi_{h}^{(-)}\right)
$$


From (101), (102), (103), and using (13),

$$
\begin{aligned}
E_{h}^{\prime \prime}\left(s_{h}\right)\left(\xi_{h}, \xi_{h}^{(+)}-\xi_{h}^{(-)}\right) \geq & \left(\lambda-c h^{1 / 2}|\log h|^{1 / 2}\|\gamma\|_{C^{3}}^{2} \nu^{2}\|s\|_{C^{2}}^{3}\right)\left\|\xi_{h}\right\|_{H^{1 / 2}}^{2} \\
& -\left\|\nabla\left(v-v_{h}\right)\right\|_{L^{2}\left(D_{h}\right)}^{2} .
\end{aligned}
$$

It remains to estimate the last term. For this it is important to notice that $\xi_{h}^{-}$ is a smooth function. We have

$$
\begin{aligned}
&\left\|\nabla\left(v-v_{h}\right)\right\|_{L^{2}\left(D_{h}\right)} \\
&=\mid\left|\Phi\left(\gamma^{\prime} \circ s \xi_{h}^{(-)}\right)-\Phi_{h} I_{h}\left(\gamma^{\prime} \circ s_{h} \xi_{h}^{(-)}\right)\right|_{H^{1}\left(D_{h}\right)} \\
& \leq \quad\left|\Phi\left(\gamma^{\prime} \circ s\left(\xi_{h}^{(-)}-\xi_{h}^{-}\right)\right)\right|_{H^{1}\left(D_{h}\right)}+\left|\Phi_{h} I_{h}\left(\gamma^{\prime} \circ s_{h}\left(\xi_{h}^{(-)}-\xi_{h}^{-}\right)\right)\right|_{H^{1}\left(D_{h}\right)} \\
&+\left|\Phi\left(\gamma^{\prime} \circ s \xi_{h}^{-}\right)-\Phi_{h} I_{h}\left(\gamma^{\prime} \circ s \xi_{h}^{-}\right)\right|_{H^{1}\left(D_{h}\right)} \\
&+\left|\Phi_{h} I_{h}\left(\left(\gamma^{\prime} \circ s-\gamma^{\prime} \circ s_{h}\right) \xi_{h}^{-}\right)\right|_{H^{1}\left(D_{h}\right)} \\
&= I_{3}+I_{4}+I_{5}+I_{6} .
\end{aligned}
$$

But

$$
\begin{aligned}
I_{3} & \leq\left|\gamma^{\prime} \circ s\left(\xi_{h}^{(-)}-\xi_{h}^{-}\right)\right|_{H^{1 / 2}} \\
& \leq\left\|\gamma^{\prime} \circ s\right\|_{C^{1}}\left\|\xi_{h}^{(-)}-\xi_{h}^{-}\right\|_{H^{1 / 2}} \\
& \leq \operatorname{ch}\|\gamma\|_{C^{2}}\|s\|_{C^{1}} \nu\left\|\xi_{h}\right\|_{H^{1 / 2}} \text { by (34). }
\end{aligned}
$$

And

$$
\begin{aligned}
I_{4} & \leq\left|\gamma^{\prime} \circ s_{h}\left(\xi_{h}^{-}-\xi_{h}^{(-)}\right)\right|_{H^{1 / 2}}+c h^{1 / 2}\left|\gamma^{\prime} \circ s_{h}\left(\xi_{h}^{-}-\xi_{h}^{(-)}\right)\right|_{H^{1}} \quad \text { by (65) } \\
& \leq c\left\|\gamma^{\prime} \circ s_{h}\right\|_{C^{1}}\left(\left\|\xi_{h}^{-}-\xi_{h}^{(-)}\right\|_{H^{1 / 2}}+h^{1 / 2}\left\|\xi_{h}^{-}-\xi_{h}^{(-)}\right\|_{H^{1}}\right) \\
& \leq c h\|\gamma\|_{C^{2}}\|s\|_{C^{1}} \nu\left\|\xi_{h}\right\|_{H^{1 / 2}} \quad \text { from (34). }
\end{aligned}
$$

Also

$$
\begin{aligned}
I_{5} & \leq c h^{1 / 2}\left|\gamma^{\prime} \circ s \xi_{h}^{-}\right|_{H^{1}} \quad \text { by }(64) \\
& \leq\left. c h^{1 / 2}\left\|\gamma^{\prime} \circ s\right\|_{C^{1}} \xi_{h}^{-}\right|_{H^{1}} \\
& \leq c h^{1 / 2}\|\gamma\|_{C^{2}}\|s\|_{C^{1}} \nu\left\|\xi_{h}\right\|_{H^{1 / 2}} \quad \text { by }(12) .
\end{aligned}
$$

Finally,

$$
\begin{aligned}
I_{6} & \leq c\left|\left(\gamma^{\prime} \circ s-\gamma^{\prime} \circ s_{h}\right) \xi_{h}^{-}\right|_{H^{1 / 2}}+c h^{1 / 2}\left|\left(\gamma^{\prime} \circ s-\gamma^{\prime} \circ s_{h}\right) \xi_{h}^{-}\right|_{H^{1}} \quad \text { by (65) } \\
& \leq c\left\|\gamma^{\prime} \circ s-\gamma^{\prime} \circ s_{h}\right\|_{C^{0,1}}\left(\left\|\xi_{h}^{-}\right\|_{H^{1 / 2}}+h^{1 / 2}\left\|\xi_{h}^{-}\right\|_{H^{1}}\right) \quad \text { by }(49) \text { and (51) } \\
& \leq c\|\gamma\|_{C^{3}}\|s\|_{C^{1}}\left\|s-s_{h}\right\|_{C^{0,1}} \nu\left\|\xi_{h}\right\|_{H^{1 / 2}} \quad \text { by }(63) \text { and (12) } \\
& \leq c h\|\gamma\|_{C^{3}}\|s\|_{C^{2}}^{2} \nu\left\|\xi_{h}\right\|_{H^{1 / 2}} \quad \text { from (23). }
\end{aligned}
$$

If we now put together the estimates for $I_{3}, I_{4}, I_{5}$ and $I_{6}$, we get

$$
\left\|\nabla\left(v-v_{h}\right)\right\|_{L^{2}\left(D_{h}\right)} \leq c h^{1 / 2}\|\gamma\|_{C^{3}} \nu\|s\|_{C^{2}}^{2}\left\|\xi_{h}\right\|_{H^{1 / 2}} .
$$

This together with (105) proves the proposition.

The next result gives the main error estimates for discrete maps $s_{h}$ which are stationary for $E_{h}$. As remarked at the beginning of this section, the proof uses Lemma 5.1. The necessary estimates were established in Propositions 4.2, 5.2 and 5.3. 
Theorem 5.4. Assume $\gamma \in C^{4}$. Let $s$ be a monotone nondegenerate stationary point for $E$, with nondegeneracy constant $\lambda$ as in (13).

Then there exist positive constants $h_{0}$ and $c_{0}$ depending on $\|\gamma\|_{C^{4}}$ and $\left\|\left|\gamma^{\prime}\right|^{-1}\right\|_{L^{\infty}}$, and $\lambda$ in the case of $h_{0}$, such that if $0<h \leq h_{0}$ then there exists $s_{h} \in \mathcal{H}_{h}$ which is stationary for $E_{h}$ and satisfies

$$
\left\|s-s_{h}\right\|_{H^{1 / 2}} \leq c_{0} \lambda^{-1} h .
$$

Moreover, there exists $\epsilon_{0}=\epsilon_{0}\left(\|\gamma\|_{C^{4}},\left\|\left|\gamma^{\prime}\right|^{-1}\right\|_{L^{\infty}}, \lambda\right)>0$ such that $s_{h}$ is the unique stationary point for $E_{h}$ satisfying

$$
\left\|s-s_{h}\right\|_{H^{1 / 2}} \leq \epsilon_{0}|\log h|^{-1}
$$

Proof. We will apply Lemma 5.1 with $\mathcal{X}=\mathcal{H}_{h}, X=H_{h}, Y=H_{h}^{\prime}$ (the dual space of $\left.H_{h}\right), f=E_{h}^{\prime}$ and $x_{0}=p_{h} s$.

Note that

$$
E_{h}^{\prime}: \mathcal{H}_{h} \rightarrow H_{h}^{\prime}
$$

From Propositions 4.2 and 2.1, since $E^{\prime}(s)=0$,

$$
\left\|E_{h}^{\prime}\left(p_{h} s\right)\right\|_{H_{h}^{\prime}} \leq c_{1} h
$$

where $c_{1}=c_{1}\left(\|\gamma\|_{C^{3}},\left\|\left|\gamma^{\prime}\right|^{-1}\right\|_{L^{\infty}}\right)$.

The derivative $\left(E_{h}^{\prime}\right)^{\prime}$ of $E_{h}^{\prime}$ is a map

$$
\left(E_{h}^{\prime}\right)^{\prime}: \mathcal{H}_{h} \rightarrow L\left(H_{h}, H_{h}^{\prime}\right)
$$

and is naturally identified in the usual way with $E_{h}^{\prime \prime}$ via

$$
\left\langle\left\langle\left(E_{h}^{\prime}\right)^{\prime}\left(t_{h}\right), \xi_{h}\right\rangle, \eta_{h}\right\rangle=E_{h}^{\prime \prime}\left(t_{h}\right)\left(\xi_{h}, \eta_{h}\right)
$$

for all $t_{h} \in \mathcal{H}_{h}$ and $\xi_{h}, \eta_{h} \in \mathcal{H}_{h}$. From Propositions 5.3, 2.1 and 2.2, since $h^{1 / 2}|\log h|^{1 / 2} \rightarrow 0$ as $h \rightarrow 0$,

$$
E_{h}^{\prime \prime}\left(p_{h} s\right)\left(\xi_{h}, \xi_{h}^{(+)}-\xi_{h}^{(-)}\right) \geq \frac{3 \lambda}{4}\left\|\xi_{h}\right\|_{H^{1 / 2}}^{2}
$$

for all $\xi_{h} \in H_{h}$, provided $0<h \leq h_{0}=h_{0}\left(\|\gamma\|_{C^{4}},\left\|\left|\gamma^{\prime}\right|^{-1}\right\|_{L^{\infty}}, \lambda\right)$. But

$$
\begin{aligned}
\left\|\xi_{h}^{(+)}-\xi_{h}^{(-)}\right\|_{H^{1 / 2}} & \leq\left\|\xi_{h}^{+}-\xi_{h}^{-}\right\|_{H^{1 / 2}}+\left\|\xi_{h}^{+}-\xi_{h}^{(+)}\right\|_{H^{1 / 2}}+\left\|\xi_{h}^{-}-\xi_{h}^{(-)}\right\|_{H^{1 / 2}} \\
& \leq(1+c h \nu)\left\|\xi_{h}\right\|_{H^{1 / 2}}
\end{aligned}
$$

from (36) and (34), and since $\left\|\xi_{h}^{+}-\xi_{h}^{-}\right\|_{H^{1 / 2}}=\left\|\xi_{h}\right\|_{H^{1 / 2}}$. Hence from (110), (111) and (112),

$$
\left\langle\left(E_{h}^{\prime}\right)^{\prime}\left(p_{h} s\right), \xi_{h}\right\rangle\left(\xi_{h}^{(+)}-\xi_{h}^{(-)}\right) \geq \frac{\lambda}{2}\left\|\xi_{h}\right\|_{H^{1 / 2}}\left\|\xi_{h}^{(+)}-\xi_{h}^{(-)}\right\|_{H^{1 / 2}}
$$

for all $\xi \in H_{h}$, provided $0<h \leq h_{0}$ for a new $h_{0}$ with the same dependencies as before. Thus for each $\xi_{h} \in H_{h}$ with $\left\|\xi_{h}\right\|_{H^{1 / 2}}=1$, the map $\left\langle\left(E_{h}^{\prime}\right)^{\prime}\left(p_{h} s\right), \xi_{h}\right\rangle\left(\in H_{h}^{\prime}\right)$ has norm $\geq \lambda / 2$. It follows, since $H_{h}$ and $H_{h}^{\prime}$ have equal finite dimension, that $\left(E_{h}^{\prime}\right)^{\prime}\left(p_{h} s\right)$ is invertible and

$$
\left\|\left(\left(E_{h}^{\prime}\right)^{\prime}\left(p_{h} s\right)\right)^{-1}\right\|_{L\left(H_{h}^{\prime}, H_{h}\right)} \leq\left(\frac{\lambda}{2}\right)^{-1} .
$$


Next note that from (110) and Proposition 5.2, by the symmetry of the second derivative,

$$
\begin{aligned}
& \left\|\left(E_{h}^{\prime}\right)^{\prime}\left(p_{h} s\right)-\left(E_{h}^{\prime}\right)^{\prime}\left(p_{h} s+\eta_{h}\right)\right\|_{L\left(H_{h}^{\prime}, H_{h}\right)} \\
& \quad \leq \quad c_{2}\left(1+|\log h|^{1 / 2}\left\|\eta_{h}\right\|_{H^{1 / 2}}\right)^{2}|\log h|\left\|\eta_{h}\right\|_{H^{1 / 2}}
\end{aligned}
$$

for all $\eta_{h} \in H_{h}$, where $c_{2}=c_{2}\left(\|\gamma\|_{C^{4}},\left\|\left|\gamma^{\prime}\right|^{-1}\right\|_{L^{\infty}}\right)$ from Proposition 2.1.

We can now choose $\epsilon_{0}=\epsilon_{0}\left(\|\gamma\|_{C^{4}},\left\|\left|\gamma^{\prime}\right|^{-1}\right\|_{L^{\infty}}, \lambda\right)$ so that $|\log h|\left\|\eta_{h}\right\|_{H^{1 / 2}}<\epsilon_{0}$ implies

$$
\left\|\left(E_{h}^{\prime}\right)^{\prime}\left(p_{h} s\right)-\left(E_{h}^{\prime}\right)^{\prime}\left(p_{h} s+\eta_{h}\right)\right\|_{L\left(H_{h}^{\prime}, H_{h}\right)} \leq \frac{\lambda}{4} .
$$

By further restricting $h_{0}$, again with the same dependencies, we can ensure that, see (109),

$$
0<h \leq h_{0} \quad \text { implies } \quad c_{1} h \leq \frac{\lambda}{4} \epsilon_{0}|\log h|^{-1} .
$$

From (109), (114), (115), (116) and Lemma 5.1 with $\alpha=\lambda / 2, \beta=\lambda / 4, \delta=c_{1} h$ and $\epsilon=\epsilon_{0}|\log h|^{-1}$, it follows that for $0<h \leq h_{0}$ there is a unique $s_{h} \in \mathcal{H}_{h}$ which is stationary for $E_{h}$ and such that

$$
\left\|s_{h}-p_{h} s\right\|_{H^{1 / 2}} \leq \epsilon_{0}|\log h|^{-1} .
$$

Next apply Lemma 5.1 with $\alpha=\lambda / 2, \beta=\lambda / 4, \delta=c_{1} h$ and $\epsilon=4 c_{1} h / \lambda$. Note that $\delta=(\alpha-\beta) \epsilon\left(=c_{1} h\right)$. Moreover, if $0<h \leq h_{0}$ then $\epsilon \leq \epsilon_{0}|\log h|^{-1}$ from (116). It follows that the unique stationary $s_{h}$ as in (117) satisfies

$$
\left\|s_{h}-p_{h} s\right\|_{H^{1 / 2}} \leq \frac{c_{0}}{\lambda} h
$$

where $c_{0}=4 c_{1}$.

Since

$$
\left\|s-p_{h} s\right\|_{H^{1 / 2}} \leq c h^{3 / 2}\|s\|_{H^{2}} \leq c h^{3 / 2}\|s\|_{C^{2}} \leq c h^{3 / 2}\|\gamma\|_{C^{3}},
$$

we may replace $p_{h} s$ by $s$ in (117) and (118), after further restricting $h_{0}, \epsilon_{0}$ and $c_{0}$ if necessary.

Remark. Suppose $u=\Phi(\gamma \circ s): D \rightarrow \Re^{n}$ is harmonic. Then

$$
\begin{aligned}
\|u\|_{H^{1}\left(D \backslash D_{h}\right)} & \leq c h\|u\|_{H^{1}(\partial D)} \quad \text { from (70) and (71) } \\
& \leq c h\|\gamma\|_{C^{1}}\|s\|_{C^{1}} \text { from (57) and (59) } \\
& \leq c h,
\end{aligned}
$$

where $c=c\left(\|\gamma\|_{C^{2}}\right)$. Thus the contribution to $\|u\|_{H^{1}(D)}$ from the boundary strip $D \backslash D_{h}$ is $O(h)$. This is consistent with the order of approximation in the following theorem.

Theorem 5.5. Assume $\gamma \in C^{4}$. Let $u$ be a nondegenerate minimal surface spanning $\Gamma$ with nondegeneracy constant $\lambda$ as in (13).

Then there exist positive constants $h_{0}$ and $c_{0}$ depending on $\|\gamma\|_{C^{4}}$ and $\left\|\left|\gamma^{\prime}\right|^{-1}\right\|_{L^{\infty}}$, and $\lambda$ in the case of $h_{0}$, such that if $0<h \leq h_{0}$, then there is a discrete minimal surface $u_{h}$ satisfying

$$
\left\|u-u_{h}\right\|_{H^{1}\left(D_{h}\right)} \leq c_{0} \lambda^{-1} h .
$$


Moreover, there exists $\epsilon_{0}=\epsilon_{0}\left(\|\gamma\|_{C^{4}},\left\|\left|\gamma^{\prime}\right|^{-1}\right\|_{L^{\infty}}, \lambda\right)>0$ such that if $u=\Phi(\gamma \circ s)$ and $u_{h}=\Phi_{h} I_{h}\left(\gamma \circ s_{h}\right)$, then $u_{h}$ is the unique discrete minimal surface satisfying

$$
\left\|s-s_{h}\right\|_{H^{1 / 2}} \leq \epsilon_{0}|\log h|^{-1} .
$$

Proof. Let

$$
u=\Phi(\gamma \circ s), \quad u_{h}=\Phi_{h} I_{h}\left(\gamma \circ s_{h}\right),
$$

where $s$ and $s_{h}$ are as in Proposition 5.4. We begin with $h_{0}, \epsilon_{0}$ and $c_{0}$ as in Proposition 5.4.

Now

$$
\begin{aligned}
\mid u & -\left.u_{h}\right|_{H^{1}\left(D_{h}\right)} \\
& =\left|\Phi(\gamma \circ s)-\Phi_{h} I_{h}\left(\gamma \circ s_{h}\right)\right|_{H^{1}\left(D_{h}\right)} \\
& \leq\left|\Phi(\gamma \circ s)-\Phi_{h} I_{h}\left(\gamma \circ p_{h} s\right)\right|_{H^{1}\left(D_{h}\right)}+\left|\Phi_{h} I_{h}\left(\gamma \circ p_{h} s-\gamma \circ s_{h}\right)\right|_{H^{1}\left(D_{h}\right)} \\
(121) & =A+B .
\end{aligned}
$$

But

$$
A \leq c h\|\gamma\|_{C^{2}}\|s\|_{C^{2}}^{2},
$$

by the estimate (77) for $I_{1}$ in the proof of Proposition 4.1.

Also,

$$
B \leq c\left(1+|\log h|^{1 / 2}\left\|s_{h}-p_{h} s\right\|_{H^{1 / 2}}\right)\left\|s_{h}-p_{h} s\right\|_{H^{1 / 2}}\|\gamma\|_{C^{2}}\|s\|_{C^{1}}
$$

by the estimate for $A_{1}$ in the proof of Proposition 5.2, with $s_{h}$ and $\eta_{h}$ there replaced by $p_{h} s$ and $s_{h}-p_{h} s$, respectively. Hence

$$
B \leq \frac{c_{0}}{\lambda} h \quad \text { from (118) }
$$

provided $0<h \leq h_{0}$, for a new $h_{0}$ and $c_{0}$ with the same dependencies.

It follows from (121), (122) and (123), possibly again with new $c_{0}$ and $h_{0}$ with the same dependencies as before, that

$$
\left|u-u_{h}\right|_{H^{1}\left(D_{h}\right)} \leq \frac{c_{0}}{\lambda} h
$$

if $0<h \leq h_{0}$.

Now

$$
\left\|u-u_{h}\right\|_{H^{1}\left(D_{h}\right)} \leq c\left(\left|u-u_{h}\right|_{H^{1}\left(D_{h}\right)}+\left\|u-u_{h}\right\|_{L^{2}\left(\partial D_{h}\right)}\right),
$$

as follows easily by integrating along rays ( $c$ depends only on $n)$. It is routine to estimate $\left\|u-u_{h}\right\|_{L^{2}\left(\partial D_{h}\right)}$. Let

$$
\tilde{u}_{h}=\Phi_{h} I_{h}\left(\gamma \circ p_{h} s\right)
$$

Then

$$
\begin{aligned}
\left\|u-u_{h}\right\|_{L^{2}\left(\partial D_{h}\right)} & \leq\left\|u-\tilde{u}_{h}\right\|_{L^{2}\left(\partial D_{h}\right)}+\left\|\tilde{u}_{h}-u_{h}\right\|_{L^{2}\left(\partial D_{h}\right)} \\
& \leq c h^{2}\|\gamma\|_{C^{2}}\|s\|_{C^{2}}^{2}+\left\|\tilde{u}_{h}-u_{h}\right\|_{L^{2}\left(\partial D_{h}\right)} \\
& =c h^{2}\|\gamma\|_{C^{2}}\|s\|_{C^{2}}^{2}+D
\end{aligned}
$$


from the argument used to estimate $I_{2}$ in the proof of Proposition 4.1, with $u_{h}$ there replaced by $\tilde{u}_{h}$. Moreover,

$$
\begin{aligned}
D & =\left\|I_{h}\left(\gamma \circ p_{h} s-\gamma \circ s_{h}\right)\right\|_{L^{2}\left(\partial D_{h}\right)} \\
& \leq\left\|I_{h}^{\partial D}\left(\gamma \circ p_{h} s-\gamma \circ s_{h}\right)\right\|_{L^{2}(\partial D)} \quad \text { by }(21) \text { as }|\nabla \pi| \leq 1 \\
& \leq\left\|\gamma \circ p_{h} s-\gamma \circ s_{h}\right\|_{L^{2}}+c h\left\|\gamma \circ p_{h} s-\gamma \circ s_{h}\right\|_{H^{1}} \\
& \leq c\|\gamma\|_{C^{1}}\left\|p_{h} s-s_{h}\right\|_{L^{2}}+c h\|\gamma\|_{C^{2}}\|s\|_{C^{1}}\left\|p_{h} s-s_{h}\right\|_{H^{1}} \\
& \quad \text { by }(60),(62) \text { and }(23) \\
& \leq c\|\gamma\|_{C^{2}}\|s\|_{C^{1}}\left\|p_{h} s-s_{h}\right\|_{L^{2}} \quad \text { by a standard inverse estimate } \\
& \leq \frac{c_{0}}{\lambda} h \text { from (118) }
\end{aligned}
$$

with a new $c_{0}$ but with the same dependencies, for $0<h \leq h_{0}$. From (124), (125), (126) and (127) it follows that if $0<h \leq h_{0}$ then

$$
\left\|u-u_{h}\right\|_{H^{1}\left(D_{h}\right)} \leq \frac{c_{0}}{\lambda} h,
$$

where $c_{0}=c_{0}\left(\|\gamma\|_{C^{3}},\left\|\left|\gamma^{\prime}\right|^{-1}\right\|_{L^{\infty}}\right)$ and $h_{0}=h_{0}\left(\|\gamma\|_{C^{3}},\left\|\left|\gamma^{\prime}\right|^{-1}\right\|_{L^{\infty}}, \lambda\right)$.

\section{ACKNowledgments}

The first author would like to thank the Centre for Mathematics and its Applications, and the second author would like to thank the Institut für Angewandte Mathematik (Universität Freiberg), for their hospitality during the course of this work. The second author would also like to thank Gaven Martin for helpful discussions on trace theory and related matters. This research has been partially supported by the Australian Research Council.

\section{REFERENCES}

[Be] M. Berger, Nonlinearity and Functional Analysis, Academic Press 1977. MR 58:7671

[BPS] J.H. Bramble, J.E. Pasciak \& A.H. Schatz, An Iterative Method for Elliptic Problems on Regions Partitioned into Substructures, Math. Comp. 46 (1986), 361-369. MR 88a:65123

[DH0] G. Dziuk, J.E. Hutchinson, On the approximation of unstable parametric minimal surfaces, preprint No. 340 (1994) SFB 256, Bonn, or CMA Math. Res. Rep. 9 (1994), Australian National University.

[DH1] G. Dziuk, J.E. Hutchinson, On the approximation of unstable parametric minimal surfaces, Calc. Var. 4 (1996), 27-58. MR 96m:49073

[DH3] G. Dziuk, J.E. Hutchinson, A Finite Element Method for the Computation of Parametric Minimal Surfaces, Tatra Mount. Math. J. 4 (1994), 49-62. MR 95g:65151

[DH4] G. Dziuk, J.E. Hutchinson, The Discrete Plateau Problem: Algorithm and Numerics, Math. Comp. 68 (1999), 1-23.

[JK] D.S. Jerison \& C.E. Kenig, Boundary Value Problems on Lipschitz Domains, Studies in Mathematics 23, 1-68, ed. W. Littman, MAA Studies in Mathematics. MR 85f:35057

[LM] J.L. Lions \& E. Magenes, Non-Homogeneous Boundary Value Problems and Applications I, Grundlehren der Mathematischen Wissenschaften 181, Springer-Verlag 1972. MR 50:2670

[St] M. Struwe, Plateau's Problem and the Calculus of Variations, Mathematical Notes 35, Princeton University Press 1988. MR 90h:58016 
Institut für Angewandte Mathematik, Universität Freiburg, Hermann-Herder-Str. 10, D-79104 Freiburg i. Br., GERMANY

E-mail address: gerd@mathematik.uni-freiburg.de

Department of Mathematics, School of Mathematical Sciences, Australian National University, GPO Box 4, Canberra, ACT 0200, AUStralia

E-mail address: John.Hutchinson@anu.edu.au 